\title{
Holomorphic discs and sutured manifolds
}

\begin{abstract}
ANDRÁS JUHÁSZ
In this paper we construct a Floer-homology invariant for a natural and wide class of sutured manifolds that we call balanced. This generalizes the Heegaard Floer hat theory of closed three-manifolds and links. Our invariant is unchanged under product decompositions and is zero for nontaut sutured manifolds. As an application, an invariant of Seifert surfaces is given and is computed in a few interesting cases.
\end{abstract}

57M27, 57R58

\section{Introduction}

In Ozsváth and Szabó [9] a Floer homology invariant was defined for closed oriented 3manifolds. This theory was extended to knots by Ozsváth and Szabó [8] and Rasmussen [12] and recently to links again by Ozsváth and Szabó [7]. Motivated by a conjecture that knot Floer homology detects fibred knots (Conjecture 10.3, originally proposed in [10]) and a characterization of fibred knots by Gabai [2], we extend Heegaard Floer hat theory to a class of sutured manifolds that we call balanced (Definition 2.2). This theory provides us with a new invariant that we call sutured Floer homology, in short, SFH. In particular, for every closed oriented 3-manifold $Y$ and every link $L \subset Y$ we construct balanced sutured manifolds $Y(1)$ and $Y(L)$ such that $\widehat{\mathrm{HF}}(Y)=\mathrm{SFH}(Y(1))$ and $\widehat{\operatorname{HFL}}(L)=\operatorname{SFH}(Y(L)) \otimes \mathbb{Z}_{2}$. Any group $\operatorname{SFH}(M, \gamma)$ decomposes into a direct sum along relative $\operatorname{Spin}^{c}$ structures on the sutured manifold $(M, \gamma)$ and each summand possesses a relative grading.

To construct the invariant we define the notion of a balanced Heegaard diagram (Definition 2.11), which consists of a compact surface $\Sigma$ with no closed components and sets of curves $\boldsymbol{\alpha}$ and $\boldsymbol{\beta}$ of the same cardinality $d$ that are also linearly independent in $H_{1}(\Sigma ; \mathbb{Q})$. These data provide the input for the usual construction of Lagrangian Floer homology applied to $\mathbb{T}_{\alpha}, \mathbb{T}_{\beta} \subset \operatorname{Sym}^{d}(\Sigma)$.

The invariant that we have constructed is unchanged under product decompositions of sutured manifolds (Lemma 9.13) and is zero for nontaut sutured manifolds (Proposition 9.18). In the last chapter we assign to every Seifert surface $R \subset S^{3}$ a sutured manifold $S^{3}(R)$ and we compute $\operatorname{SFH}\left(S^{3}(R)\right)$ in a few cases. These computations indicate a 
relationship between the top nonzero term of knot Floer homology and sutured Floer homology of the sutured manifold obtained from a minimal genus Seifert surface. This relationship is the subject of Conjecture 10.2.

Acknowledgements I would like to thank Professor Zoltán Szabó for leading me to the idea of sutured Floer homology and for his support during the course of this work. I would also like to thank Yi Ni for the helpful discussions about the topic, for thoroughly reading the first version of this paper and for proving Proposition 9.18. I am grateful to the referee for carefully reading the manuscript and making several useful remarks. This research was partially supported by OTKA grant no. T49449.

\section{Heegaard diagrams of sutured manifolds}

First we recall the notion of a sutured manifold as defined by Gabai [1].

Definition 2.1 A sutured manifold $(M, \gamma)$ is a compact oriented 3-manifold $M$ with boundary together with a set $\gamma \subset \partial M$ of pairwise disjoint annuli $A(\gamma)$ and tori $T(\gamma)$. Furthermore, the interior of each component of $A(\gamma)$ contains a suture, ie, a homologically nontrivial oriented simple closed curve. We denote the union of the sutures by $s(\gamma)$.

Finally every component of $R(\gamma)=\partial M \backslash \operatorname{Int}(\gamma)$ is oriented. Define $R_{+}(\gamma)$ (or $\left.R_{-}(\gamma)\right)$ to be those components of $\partial M \backslash \operatorname{Int}(\gamma)$ whose normal vectors point out of (into) $M$. The orientation on $R(\gamma)$ must be coherent with respect to $s(\gamma)$, ie, if $\delta$ is a component of $\partial R(\gamma)$ and is given the boundary orientation, then $\delta$ must represent the same homology class in $H_{1}(\gamma)$ as some suture.

In this paper we will restrict our attention to a special class of sutured manifolds.

Definition 2.2 A balanced sutured manifold is a sutured manifold $(M, \gamma)$ such that $\mathrm{M}$ has no closed components, $\chi\left(R_{+}(\gamma)\right)=\chi\left(R_{-}(\gamma)\right)$, and the map from $\pi_{0}(A(\gamma))$ to $\pi_{0}(\partial M)$ is surjective.

Note that the last condition implies that for a balanced sutured manifold $T(\gamma)=\varnothing$. A balanced sutured manifold is completely determined by $M$ and $s(\gamma)$. Therefore, one can view $\gamma$ as a set of thick oriented curves in $\partial M$ where such curves induce the orientations on $\partial M \backslash \operatorname{Int}(\gamma)$. Now we list a few important examples of balanced sutured manifolds. 
Example 2.3 Let $Y$ be a closed connected oriented 3-manifold and we are also given pairwise disjoint closed 3-balls $B_{1}, \ldots, B_{k} \subset Y$. For $1 \leq i \leq k$ choose an oriented simple closed curve $s_{i} \subset \partial B_{i}$ together with a regular neighborhood $\gamma_{i}=N\left(s_{i}\right)$. If

$$
M=Y \backslash \bigcup_{i=1}^{k} \operatorname{Int}\left(B_{i}\right) \text { and } \gamma=\bigcup_{i=1}^{k} \gamma_{i}
$$

then the pair $(M, \gamma)$ defines a balanced sutured manifold with sutures $s(\gamma)=\bigcup_{i=1}^{k} s_{i}$. The sutured manifold $(M, \gamma)$ only depends on $Y$ and $k$, we denote it by $Y(k)$. Note that $Y(k)$ uniquely determines $Y$.

If $(N, v)$ is a connected balanced sutured manifold then let $N(k)$ denote the connected sum $(N, v) \# S^{3}(k)$. This is also a balanced sutured manifold.

Example 2.4 Let $L \subset Y$ be a link of $k$ components in a closed connected oriented 3-manifold $Y$. Choose a closed regular neighborhood $N(L)$ of $L$. For every component $L_{i}$ of $L(1 \leq i \leq k)$ take two meridians $s_{i}$ and $s_{i}^{\prime}$ of $L_{i}$ oppositely oriented, that is, $\left[s_{i}\right]=-\left[s_{i}^{\prime}\right]$ in $H_{1}\left(\partial N\left(L_{i}\right) ; \mathbb{Z}\right)$. Choose regular neighborhoods $\gamma_{i}=N\left(s_{i}\right)$ and $\gamma_{i}^{\prime}=N\left(s_{i}^{\prime}\right)$ in $\partial N\left(L_{i}\right)$ and let $\gamma=\bigcup_{i=1}^{k}\left(\gamma_{i} \cup \gamma_{i}^{\prime}\right)$; furthermore let $M=Y \backslash \bigcup_{i=1}^{k} \operatorname{Int}\left(N\left(L_{i}\right)\right)$. This way we obtain a balanced sutured manifold $(M, \gamma)$. We can reconstruct $L$ from $(M, \gamma)$ using Dehn filling as follows. For each component $T_{i}^{2}$ of $\partial M$ glue in a solid torus $S^{1} \times D^{2}$ so that $\{1\} \times \partial D^{2}$ maps to one component of $s(\gamma) \cap T_{i}^{2}$, let $L_{i}$ be the image of $S^{1} \times\{0\}$. Note that if we choose the other component of $s(\gamma) \cap T_{i}^{2}$ only the orientation of $L_{i}$ changes, and choosing different images for the longitude $S^{1} \times\{1\}$ corresponds to choosing different framings of $L_{i}$.

$(M, \gamma)$ is uniquely determined by the link $L$; let us use the notation $Y(L)$ for the sutured manifold $(M, \gamma)$. We saw above that $Y(L)$ uniquely determines $L$. If in addition we fix an ordering of the components of $s(\gamma) \cap T_{i}^{2}$ (ie, we distinguish between $s_{i}$ and $s_{i}^{\prime}$ ) we uniquely define an orientation of $L$.

The following two examples can be found in [2].

Example 2.5 Let $R$ be a compact oriented surface with no closed components. Then there is an induced orientation on $\partial R$. Let $M=R \times I$, define $\gamma=\partial R \times I$, finally put $s(\gamma)=\partial R \times\{1 / 2\}$. The balanced sutured manifold $(M, \gamma)$ obtained by this construction is called a product sutured manifold.

Example 2.6 Let $Y$ be a closed connected oriented 3-manifold and let $R \subset Y$ be a compact oriented surface with no closed components. We define $Y(R)=(M, \gamma)$ to be the sutured manifold where $M=Y \backslash \operatorname{Int}(R \times I)$, the suture $\gamma=\partial R \times I$ and $s(\gamma)=\partial R \times\{1 / 2\}$. Then $Y(R)$ is balanced. 
Next we introduce sutured Heegaard diagrams. They generalize Heegaard diagrams of closed 3-manifolds so that we can also describe sutured manifolds.

Definition 2.7 A sutured Heegaard diagram is a tuple $(\Sigma, \boldsymbol{\alpha}, \boldsymbol{\beta})$, where $\Sigma$ is a compact oriented surface with boundary and $\boldsymbol{\alpha}=\left\{\alpha_{1}, \ldots, \alpha_{m}\right\}$ and $\boldsymbol{\beta}=\left\{\beta_{1}, \ldots, \beta_{n}\right\}$ are two sets of pairwise disjoint simple closed curves in $\operatorname{Int}(\Sigma)$.

Definition 2.8 Every sutured Heegaard diagram $(\Sigma, \boldsymbol{\alpha}, \boldsymbol{\beta})$ uniquely defines a sutured manifold $(M, \gamma)$ using the following construction.

Let $M$ be the 3-manifold obtained from $\Sigma \times I$ by attaching 3-dimensional 2-handles along the curves $\alpha_{i} \times\{0\}$ and $\beta_{j} \times\{1\}$ for $i=1, \ldots, m$ and $j=1, \ldots, n$. The sutures are defined by taking $\gamma=\partial M \times I$ and $s(\gamma)=\partial M \times\{1 / 2\}$.

Proposition 2.9 If $(M, \gamma)$ is defined by $(\Sigma, \boldsymbol{\alpha}, \boldsymbol{\beta})$ then $(M, \gamma)$ is balanced if and only if $|\boldsymbol{\alpha}|=|\boldsymbol{\beta}|$ and the maps $\pi_{0}(\partial \Sigma) \rightarrow \pi_{0}(\Sigma \backslash \bigcup \boldsymbol{\alpha})$ and $\pi_{0}(\partial \Sigma) \rightarrow \pi_{0}(\Sigma \backslash \bigcup \boldsymbol{\beta})$ are surjective. The second condition is equivalent to saying that $\Sigma$ has no closed components and the elements of $\boldsymbol{\alpha}$ and $\boldsymbol{\beta}$ are both linearly independent in $H_{1}(\Sigma ; \mathbb{Q})$.

Proof Since adding a 2-handle increases the Euler characteristics of the boundary by 2 (the boundary undergoes surgery along the attaching circle) we get the equalities $\chi\left(R_{+}(\gamma)\right)=\chi(\Sigma)+2 m$ and $\chi\left(R_{-}(\gamma)\right)=\chi(\Sigma)+2 n$. Thus $\chi\left(R_{+}(\gamma)\right)=\chi\left(R_{-}(\gamma)\right)$ if and only if $|\boldsymbol{\alpha}|=|\boldsymbol{\beta}|$.

Note that every component of $\partial M$ contains a suture exactly when $R_{-}(\gamma)$ and $R_{+}(\gamma)$ have no closed components. Since $R_{-}(\gamma)$ is obtained from $\Sigma$ by performing surgery along $\boldsymbol{\alpha}$, components of $\Sigma \backslash \bigcup \boldsymbol{\alpha}$ naturally correspond to components of $R_{-}(\gamma)$. Thus a component of $\Sigma \backslash \bigcup \alpha$ contains a component of $\partial \Sigma$ if and only if the corresponding component of $R_{-}(\gamma)$ has nonempty boundary. So $R_{-}(\gamma)$ has no closed components if and only if the map $\pi_{0}(\partial \Sigma) \rightarrow \pi_{0}(\Sigma \backslash \bigcup \alpha)$ is surjective. A similar argument can be used for $R_{+}(\gamma)$.

The last statement follows from Lemma 2.10.

Lemma 2.10 Let $\Sigma$ be a compact oriented surface with boundary and let $\alpha \subset \operatorname{Int}(\Sigma)$ be a one-dimensional submanifold of $\Sigma$. Then the map $\pi_{0}(\partial \Sigma) \rightarrow \pi_{0}(\Sigma \backslash \alpha)$ is injective if and only if $\Sigma$ has no closed components and the components of $\alpha$ are linearly independent in $H_{1}(\Sigma ; \mathbb{Q})$.

Proof In this proof every homology group is to be considered with coefficients in $\mathbb{Q}$. The components of $\alpha$ are linearly independent in $H_{1}(\Sigma)$ exactly when the map 
$i_{*}: H_{1}(\alpha) \rightarrow H_{1}(\Sigma)$ induced by the embedding $i: \alpha \hookrightarrow \Sigma$ is injective. Look at the following portion of the long exact sequence of the pair $(\Sigma, \alpha)$ :

$$
0 \rightarrow H_{2}(\Sigma) \rightarrow H_{2}(\Sigma, \alpha) \rightarrow H_{1}(\alpha) \stackrel{i_{*}}{\rightarrow} H_{1}(\Sigma) .
$$

Then we see that $H_{2}(\Sigma, \alpha) \approx H_{2}(\Sigma) \oplus \operatorname{ker}\left(i_{*}\right)$. Note that $H_{2}(\Sigma)=0$ precisely when $\Sigma$ has no closed components. Let $N(\alpha)$ be a closed regular neighborhood of $\alpha$. Then by excision

$$
H_{2}(\Sigma, \alpha) \approx H_{2}(\Sigma, N(\alpha)) \approx H_{2}(\Sigma \backslash \operatorname{Int}(N(\alpha)), \partial N(\alpha)) \approx \bigoplus_{C} H_{2}(C, \partial N(\alpha) \cap C),
$$

where $C$ runs over the components of $\Sigma \backslash \operatorname{Int}(N(\alpha))$. Thus $H_{2}(\Sigma, \alpha)=0$ if and only if for every such component $C$ the group $H_{2}(C, \partial N(\alpha) \cap C)=0$, ie, when $C \cap \partial \Sigma \neq \varnothing$. Thus $H_{2}(\Sigma, \alpha)=0$ exactly when the map $\pi_{0}(\partial \Sigma) \rightarrow \pi_{0}(\Sigma \backslash \alpha)$ is injective.

Proposition 2.9 justifies the following definition.

Definition 2.11 A sutured Heegaard diagram $(\Sigma, \boldsymbol{\alpha}, \boldsymbol{\beta})$ is called balanced if $|\boldsymbol{\alpha}|=|\boldsymbol{\beta}|$ and the maps $\pi_{0}(\partial \Sigma) \rightarrow \pi_{0}(\Sigma \backslash \bigcup \alpha)$ and $\pi_{0}(\partial \Sigma) \rightarrow \pi_{0}(\Sigma \backslash \bigcup \beta)$ are surjective.

Remark 2.12 We will use the abbreviation "balanced diagram" for "balanced sutured Heegaard diagram".

Proposition 2.13 Let $(M, \gamma)$ be a sutured manifold for which the maps

$$
\pi_{0}\left(R_{+}(\gamma)\right) \rightarrow \pi_{0}(M) \text { and } \pi_{0}\left(R_{-}(\gamma)\right) \rightarrow \pi_{0}(M)
$$

are surjective. Then there exists a sutured Heegaard diagram $(\Sigma, \boldsymbol{\alpha}, \boldsymbol{\beta})$ defining it.

Proof Fix a Riemannian metric on $M$. First we construct a special Morse function $f$ on $M$ the following way. Choose a diffeomorphism $\varphi: \gamma \rightarrow s(\gamma) \times[-1,4]$ so that $\varphi(s(\gamma))=s(\gamma) \times\{3 / 2\}$ and let $p_{2}: s(\gamma) \times[-1,4] \rightarrow[-1,4]$ be the projection onto the second factor. Then we define $f \mid \gamma$ to be $p_{2} \circ \varphi$. Furthermore, let $f \mid R_{-}(\gamma) \equiv-1$ and $f \mid R_{+}(\gamma) \equiv 4$. Now take a generic extension of $f \mid \partial M$ to $M$. Then $f: M \rightarrow \mathbb{R}$ is a Morse function.

Using [5, Theorem 4.8] we can assume that $f$ is self-indexing. Applying the idea of [5, Theorem 8.1] as follows we can assume that $f$ has no index 0 and 3 critical points. Since the map $H_{0}\left(R_{-}(\gamma)\right) \rightarrow H_{0}(M)$ is surjective $H_{0}\left(M, R_{-}(\gamma)\right)=0$. Thus, using $\mathrm{CW}$ homology, we see that for every index 0 critical point of $f$ we can find an index 1 critical point so that there is exactly one gradient flow line connecting them, and they can be canceled. Indeed, since $H_{0}\left(M, R_{-}(\gamma)\right)=0$, for every index zero critical point 
$p$ there is an index one critical point $q$ such that $p$ and $q$ are connected by an odd number of gradient flow lines. But there are only two flow lines coming out of $q$, so there is exactly one trajectory connecting $p$ and $q$. During this process we do not have to change $f \mid \partial M$. Similarly, we can cancel every index 3 critical point of $f$.

Finally, let $\Sigma=f^{-1}(3 / 2)$ and let $\boldsymbol{\alpha}$ and $\boldsymbol{\beta}$ be the intersections of $\Sigma$ with the ascending and descending manifolds of the index one and two critical points of $f$ respectively. Then $(\Sigma, \boldsymbol{\alpha}, \boldsymbol{\beta})$ defines $(M, \gamma)$.

Proposition 2.14 For every balanced sutured manifold $(M, \gamma)$ there exists a balanced diagram defining it.

Proof This is a corollary of Proposition 2.9 and Proposition 2.13.

Next we will state and prove a generalization of [9, Proposition 2.2].

Proposition 2.15 If the balanced diagrams $\left(\Sigma_{0}, \boldsymbol{\alpha}_{\mathbf{0}}, \boldsymbol{\beta}_{\mathbf{0}}\right)$ and $\left(\Sigma_{1}, \boldsymbol{\alpha}_{\mathbf{1}}, \boldsymbol{\beta}_{\mathbf{1}}\right)$ define the same balanced sutured manifold $(M, \gamma)$ then they are diffeomorphic after a finite sequence of Heegaard moves.

Proof Suppose that $\alpha \subset R_{-}(\gamma)$ is a simple closed curve such that the 1 -handle attached to $M$ along $\alpha$ can be canceled by a $0-$ handle $B^{3}$. Then the curve $\alpha$ bounds the 2-disc $\partial B^{3} \cap R_{-}(\gamma)$.

Using the above observation we get that adding a canceling pair of index 0 and 1 critical points corresponds to adding a curve $\alpha$ to $\alpha$ such that after performing surgery on $\Sigma$ along $\boldsymbol{\alpha}$ (so that we obtain $R_{-}(\gamma)$ ) the image of $\alpha$ bounds a disc.

Notation 2.16 If $\gamma$ is a set of pairwise disjoint simple closed curves in the interior of a surface $\Sigma$ then $\Sigma[\gamma]$ denotes the surface obtained by surgery on $\Sigma$ along $\gamma$.

Lemma 2.17 Let $\alpha_{1}, \ldots, \alpha_{d}, \gamma$ and $\delta$ be pairwise disjoint simple closed curves in a compact oriented surface $\Sigma$ such that the image of both $\gamma$ and $\delta$ bound a disc in $\Sigma\left[\alpha_{1}, \ldots, \alpha_{d}\right]$. Suppose that $\gamma$ is not null-homologous.

Then there is an $i \in\{1, \ldots, d\}$ such that $\gamma$ is isotopic to a curve obtained by handlesliding $\alpha_{i}$ across some collection of the $\alpha_{j}$ for $j \neq i$. Moreover, the curves $\alpha_{i}$ and $\delta$ both bound discs in $\widetilde{\Sigma}=\Sigma\left[\alpha_{1}, \ldots, \alpha_{i-1}, \alpha_{i+1}, \ldots, \alpha_{d}, \gamma\right]$. 
Proof Let $D_{\gamma}$ and $D_{\delta}$ be discs bound by $\gamma$ and $\delta$ in $\Sigma^{\prime}=\Sigma\left[\alpha_{1}, \ldots, \alpha_{d}\right]$ respectively. For $i=1, \ldots, d$ let $p_{i}, q_{i} \in \Sigma^{\prime}$ be the points corresponding to the zero-sphere which replaced the circle $\alpha_{i}$. Since $\gamma$ is not null-homologous, there is an $i \in\{1, \ldots, d\}$ such that $D_{\gamma}$ separates $p_{i}$ and $q_{i}$. We can suppose without loss of generality that $i=1$ and $p_{1} \in D_{\gamma}$ while $q_{1} \notin D_{\gamma}$. An isotopy in $D_{\gamma}$ of a small circle around $p_{1}$ to $\gamma$ corresponds to handlesliding $\alpha_{1}$ across some collection of the $\alpha_{j}$ for $j \neq 1$ so that we obtain $\gamma$.

Observe that $\widetilde{\Sigma}$ is obtained from $\Sigma^{\prime}$ by adding a tube $T$ to $\Sigma^{\prime} \backslash\left\{p_{1}, q_{1}\right\}$ and performing surgery along $\gamma$. We take $\Sigma^{\prime} \backslash \gamma$ and pinch the boundary component corresponding to $\partial D_{\gamma}$ to $p_{0}$ and $\partial\left(\Sigma^{\prime} \backslash D_{\gamma}\right)$ to $q_{0}$. Then $\alpha_{1}$ is the boundary of the $\operatorname{disc}\left(D_{\gamma} \backslash\left\{p_{1}\right\}\right) \cup\left\{p_{0}\right\} \subset \widetilde{\Sigma}$.

We are now going to prove that $\delta$ bounds a disc in $\widetilde{\Sigma}$. If $p_{1} \notin D_{\delta}$ and $q_{1} \notin D_{\delta}$ then since $\delta \cap \gamma=\varnothing$ the disc $D_{\delta}$ "survives" in $\widetilde{\Sigma}$. If $p_{1} \in D_{\delta}$ and $q_{1} \notin D_{\delta}$ then in $\widetilde{\Sigma}$ the curve $\delta$ bounds $\left(D_{\gamma} \backslash D_{\delta}\right) \cup\left\{p_{0}\right\}$ if $D_{\gamma} \supset D_{\delta}$ and $\left(D_{\delta} \backslash D_{\gamma}\right) \cup\left\{q_{0}\right\}$ otherwise. If $p_{1} \notin D_{\delta}$ and $q_{1} \in D_{\delta}$ then $\delta$ bounds in $\widetilde{\Sigma}$ the disc $\left(D_{\delta} \backslash\left\{q_{1}\right\}\right) \cup T \cup\left(D_{\gamma} \backslash\left\{p_{1}\right\}\right) \cup\left\{p_{0}\right\}$. Finally, if $p_{1}, q_{1} \in D_{\delta}$ then of course $D_{\gamma} \subset D_{\delta}$, and in $\widetilde{\Sigma}$ the curve $\delta$ bounds $\left(D_{\delta} \backslash\left(D_{\gamma} \cup\left\{q_{1}\right\}\right)\right) \cup T \cup\left(D_{\gamma} \backslash\left\{p_{1}\right\}\right) \cup\left\{p_{0}, q_{0}\right\}$.

(In fact, $\Sigma^{\prime}=\Sigma\left[\alpha_{2}, \ldots, \alpha_{d}\right]\left[\alpha_{1}\right]$ and $\widetilde{\Sigma}=\Sigma\left[\alpha_{2}, \ldots, \alpha_{d}\right][\gamma]$, and furthermore the curves $\alpha_{1}$ and $\gamma$ are isotopic in $\Sigma\left[\alpha_{2}, \ldots, \alpha_{d}\right]$.)

Lemma 2.18 Let $\delta$ be a set of pairwise disjoint simple closed curves in $\Sigma$, and suppose that we are given two subsets of curves $\boldsymbol{\alpha}, \boldsymbol{\gamma} \subset \boldsymbol{\delta}$ that are linearly independent in $H_{1}(\Sigma ; \mathbb{Q})$. Suppose furthermore that the image of every $\delta \in \delta \backslash \boldsymbol{\alpha}$ bounds a disc in $\Sigma[\alpha]$. Then $\gamma$ can be obtained from $\alpha$ by a series of isotopies and handleslides. Moreover, the image of every $\delta \in \delta \backslash \boldsymbol{\gamma}$ bounds a disc in $\Sigma[\boldsymbol{\gamma}]$.

Proof Let $d=|\boldsymbol{\alpha}|=|\boldsymbol{\gamma}|$. We prove the claim using induction on $d$. The case $d=0$ is trivial. Note that it follows from the hypothesis that $\boldsymbol{\alpha}$ and $\boldsymbol{\gamma}$ span the same subspace in $H_{1}(\Sigma ; \mathbb{Q})$.

If $\boldsymbol{\alpha} \cap \boldsymbol{\gamma} \neq \varnothing$, say the curve $\alpha$ lies in the intersection, then perform surgery on $\Sigma$ along $\alpha$ to obtain a new surface $\Sigma^{\prime}$ with two marked points $p, q$ and two $(d-1)$-tuples of curves $\boldsymbol{\alpha}^{\prime}$ and $\boldsymbol{\gamma}^{\prime}$. Let $\boldsymbol{\delta}^{\prime}=\boldsymbol{\delta} \backslash\{\alpha\}$. Note that every $\delta \in \boldsymbol{\delta}^{\prime} \backslash \boldsymbol{\alpha}^{\prime}=\boldsymbol{\delta} \backslash \boldsymbol{\alpha}$ bounds a disc in $\Sigma^{\prime}\left[\boldsymbol{\alpha}^{\prime}\right]=\Sigma[\boldsymbol{\alpha}]$. Using the induction hypothesis $\boldsymbol{\alpha}^{\prime}$ and $\boldsymbol{\gamma}^{\prime}$ are related by isotopies and handleslides. We can arrange (using isotopies) that each handleslide is disjoint from $p$ and $q$. Each isotopy of a curve in $\Sigma^{\prime}$ that crosses $p$ or $q$ corresponds to a handleslide in $\Sigma$ across $\alpha$. Thus $\boldsymbol{\alpha}$ and $\boldsymbol{\gamma}$ are also related by isotopies and handleslides. Also from the induction hypothesis we get that every $\delta \in \delta^{\prime} \backslash \boldsymbol{\gamma}^{\prime}$ bounds a disc in $\Sigma^{\prime}\left[\boldsymbol{\gamma}^{\prime}\right]=\Sigma[\boldsymbol{\gamma}]$. This implies that the image of every $\delta \in \delta \backslash \boldsymbol{\gamma}$ bounds a disc in $\Sigma[\boldsymbol{\gamma}]$. 
If $\boldsymbol{\alpha} \cap \boldsymbol{\gamma}=\varnothing$ then take any $\gamma \in \boldsymbol{\gamma}$. Since elements of $\boldsymbol{\gamma}$ are linearly independent $\gamma$ is not null-homologous. Thus, using Lemma 2.17, $\gamma$ can be obtained by handlesliding some $\alpha_{i}$ across a collection of the $\alpha_{j}$ for $j \neq i$. So we have reduced to the case where the two subsets are not disjoint.

For $i \in\{0,1\}$ choose a Morse function $f_{i}$ inducing $\left(\Sigma_{i}, \boldsymbol{\alpha}_{\boldsymbol{i}}, \boldsymbol{\beta}_{\boldsymbol{i}}\right)$ as in the proof of Proposition 2.13 and let $\left\{f_{t}: 0 \leq t \leq 1\right\}$ be a generic one-parameter family of functions connecting them. We can suppose that $f_{t}$ is fixed in a neighborhood of $\partial M$. Also equip $Y$ with a generic Riemannian metric. Then there is a finite subset $E \subset I$ such that for $t \in I \backslash E$ the function $f_{t}$ is Morse with gradient flow lines flowing only from larger to strictly smaller index critical points, and thus induces a diagram $\left(\Sigma_{t}, \boldsymbol{\alpha}_{\boldsymbol{t}}, \boldsymbol{\beta}_{\boldsymbol{t}}\right)$. Here $\boldsymbol{\alpha}_{\boldsymbol{t}}$ and $\boldsymbol{\beta}_{\boldsymbol{t}}$ are the intersections of $\Sigma_{t}$ with the ascending and descending manifolds of the index one and two critical points of $f_{t}$ respectively. As $t$ passes through an element $e \in E$ the diagram corresponding to $f_{t}$ experiences one of the following changes. There is either a handleslide among the $\alpha$ curves or the $\beta$ curves (corresponding to a gradient flow line connecting two index one or two index two critical points of $f_{e}$ ), or a stabilization/destabilization (corresponding to creation/cancellation of index 1 and 2 critical points), or a new $\alpha$ or $\beta$ curve appears/disappears (corresponding to canceling index 0 and 1, or index 2 and 3 critical points). The last case is called a pair creation/cancellation.

For each $t \in I \backslash E$ choose two maximal homologically linearly independent subsets $\boldsymbol{\alpha}_{\boldsymbol{t}}^{\prime} \subset \boldsymbol{\alpha}_{\boldsymbol{t}}$ and $\boldsymbol{\beta}_{\boldsymbol{t}}^{\prime} \subset \boldsymbol{\beta}_{\boldsymbol{t}}$ that change continuously in $t$. Then of course $\boldsymbol{\alpha}_{\boldsymbol{i}}^{\prime}=\boldsymbol{\alpha}_{\boldsymbol{i}}$ and $\boldsymbol{\beta}_{\boldsymbol{i}}^{\prime}=\boldsymbol{\beta}_{\boldsymbol{i}}$ for $i=0,1$. So it is enough to show that for every $e \in E$ and sufficiently small $\varepsilon$ the diagrams $\left(\Sigma_{e-\varepsilon}, \boldsymbol{\alpha}_{\boldsymbol{e}-\boldsymbol{\varepsilon}}^{\prime}, \boldsymbol{\beta}_{\boldsymbol{e}-\boldsymbol{\varepsilon}}^{\prime}\right)$ and $\left(\Sigma_{e+\varepsilon}, \boldsymbol{\alpha}_{\boldsymbol{e}+\boldsymbol{\varepsilon}}^{\prime}, \boldsymbol{\beta}_{\boldsymbol{e}+\boldsymbol{\varepsilon}}^{\prime}\right)$ are related by isotopies, handleslides, stabilization and destabilization.

In order to do this we also need to prove the fact that for every $t \in I \backslash E$ and every curve $\alpha \in \boldsymbol{\alpha}_{\boldsymbol{t}} \backslash \boldsymbol{\alpha}_{\boldsymbol{t}}^{\prime}$ the image of $\alpha$ bounds a disc in the surface $\Sigma_{t}\left[\boldsymbol{\alpha}_{\boldsymbol{t}}^{\prime}\right]$. We prove this by induction on the component of $I \backslash E$ containing $t$. It is obviously true for $t=0$.

First consider the case when $e$ does not correspond to stabilization or destabilization. Let $\delta=\alpha_{e-\varepsilon} \cup \alpha_{e+\varepsilon}$, this is a set of pairwise disjoint curves. Furthermore, let $\alpha=\alpha_{e-\varepsilon}^{\prime}$ and $\gamma=\alpha_{e+\varepsilon}^{\prime}$. Observe that $\delta \backslash \alpha_{e-\varepsilon}$ consists of at most one curve $\delta$ obtained from either a handleslide within $\alpha_{e-\varepsilon}$ or a pair creation. Using the induction hypothesis for $t=e-\varepsilon$ we see that $\delta$ also bounds a disc in $\Sigma_{e-\varepsilon}[\boldsymbol{\alpha}]$. Thus we can apply Lemma 2.18 to $\boldsymbol{\alpha}, \boldsymbol{\gamma} \subset \boldsymbol{\delta}$ showing that $\boldsymbol{\alpha}$ and $\boldsymbol{\gamma}$ are related by isotopies and handleslides and that the induction hypothesis also holds for $t=e+\varepsilon$. A similar argument applies to the $\beta$ curves. 
Now suppose that $e$ corresponds to a stabilization; the new curves appearing are $\alpha$ and $\beta$. Define $\boldsymbol{\alpha}=\boldsymbol{\alpha}_{\boldsymbol{e}-\boldsymbol{\varepsilon}}^{\prime} \cup\{\alpha\}$ and $\boldsymbol{\beta}=\boldsymbol{\beta}_{\boldsymbol{e}-\boldsymbol{\varepsilon}}^{\prime} \cup\{\beta\}$, considered as sets of curves in $\Sigma_{\boldsymbol{e}+\varepsilon}$. Then we can apply Lemma 2.18 to $\boldsymbol{\alpha}, \boldsymbol{\alpha}_{\boldsymbol{e}+\boldsymbol{\varepsilon}}^{\prime} \subset \boldsymbol{\alpha}_{\boldsymbol{e}+\boldsymbol{\varepsilon}}$ and $\boldsymbol{\beta}, \boldsymbol{\beta}_{\boldsymbol{e}+\boldsymbol{\varepsilon}}^{\prime} \subset \boldsymbol{\beta}_{\boldsymbol{e}+\boldsymbol{\varepsilon}}$.

The case of a destabilization is proved in a similar way, by taking $\alpha=\alpha_{\boldsymbol{e}+\boldsymbol{\varepsilon}}^{\prime} \cup\{\alpha\}$ and $\boldsymbol{\beta}=\boldsymbol{\beta}_{\boldsymbol{e}+\boldsymbol{\varepsilon}}^{\prime} \cup\{\beta\}$, where $\alpha$ and $\beta$ are the curves that vanish.

Remark 2.19 From Proposition 2.15 we see that if we associate to every balanced diagram a quantity that is unchanged by isotopies, handleslides and stabilization we get a topological invariant of sutured 3-manifolds.

\section{Whitney discs and their domains}

For a surface $\Sigma$ let $\operatorname{Sym}^{d}(\Sigma)$ denote the d-fold symmetric product $\Sigma^{\times d} / S_{d}$. This is always a smooth $2 d$-manifold. A complex structure $\mathfrak{j}$ on $\Sigma$ naturally endows $\operatorname{Sym}^{d}(\Sigma)$ with a complex structure, denoted $\operatorname{Sym}^{d}(\mathrm{j})$. This structure $\operatorname{Sym}^{d}(\mathrm{j})$ is specified by the property that the quotient map $\Sigma^{d} \rightarrow \operatorname{Sym}^{d}(\Sigma)$ is holomorphic.

Definition 3.1 Let $(\Sigma, \boldsymbol{\alpha}, \boldsymbol{\beta})$ be a balanced diagram, where $\boldsymbol{\alpha}=\left\{\alpha_{1}, \ldots, \alpha_{d}\right\}$ and $\boldsymbol{\beta}=\left\{\beta_{1}, \ldots, \beta_{d}\right\}$. Then let $\mathbb{T}_{\alpha}=\left(\alpha_{1} \times \cdots \times \alpha_{d}\right) / S_{d}$ and $\mathbb{T}_{\beta}=\left(\beta_{1} \times \cdots \times \beta_{d}\right) / S_{d}$. These are $d$-dimensional tori in $\operatorname{Sym}^{d}(\Sigma)$.

Lemma 3.2 For a balanced diagram $(\Sigma, \boldsymbol{\alpha}, \boldsymbol{\beta})$ and an arbitrary complex structure j on $\Sigma$, the submanifolds $\mathbb{T}_{\alpha}, \mathbb{T}_{\beta} \subset \operatorname{Sym}^{d}(\Sigma)$ are totally real with respect to $\operatorname{Sym}^{d}(\mathrm{j})$.

Proof The submanifold $\alpha_{1} \times \cdots \times \alpha_{d} \subset \Sigma^{\times d}$ is totally real with respect to $\mathrm{j}^{\times d}$ and misses the diagonal (consisting of those $d$-tuples for which at least two coordinates coincide). The claim thus follows since the projection map $\pi: \Sigma^{\times d} \rightarrow \operatorname{Sym}^{d}(\Sigma)$ is a holomorphic local diffeomorphism away from the diagonal.

Note that if every $\alpha \in \boldsymbol{\alpha}$ and $\beta \in \boldsymbol{\beta}$ are transversal then the tori $\mathbb{T}_{\alpha}$ and $\mathbb{T}_{\beta}$ intersect transversally.

Notation 3.3 Let $\mathbb{D}$ denote the unit disc in $\mathbb{C}$, and let $e_{1}=\{z \in \partial \mathbb{D}: \operatorname{Re}(z) \geq 0\}$ and $e_{2}=\{z \in \partial \mathbb{D}: \operatorname{Re}(z) \leq 0\}$.

Definition 3.4 Let $\mathbf{x}, \mathbf{y} \in \mathbb{T}_{\alpha} \cap \mathbb{T}_{\beta}$ be intersection points. A Whitney disc connecting $\mathbf{x}$ to $\mathbf{y}$ is a continuous map $u: \mathbb{D} \rightarrow \operatorname{Sym}^{d}(\Sigma)$ such that $u(-i)=\mathbf{x}, u(i)=\mathbf{y}$ and $u\left(e_{1}\right) \subset \mathbb{T}_{\alpha}, u\left(e_{2}\right) \subset \mathbb{T}_{\beta}$. Let $\pi_{2}(\mathbf{x}, \mathbf{y})$ denote the set of homotopy classes of Whitney discs connecting $\mathbf{x}$ to $\mathbf{y}$.

Algebraic $8 \mathcal{G}$ Geometric Topology, Volume 6 (2006) 
Definition 3.5 For $z \in \Sigma \backslash(\bigcup \boldsymbol{\alpha} \cup \bigcup \boldsymbol{\beta})$ and $u$ a Whitney disc, choose a Whitney disc $u^{\prime}$ homotopic to $u$ such that $u^{\prime}$ intersects the hypersurface $\{z\} \times \operatorname{Sym}^{d-1}(\Sigma)$ transversally. Define $n_{z}(u)$ to be the algebraic intersection number $u^{\prime} \cap\left(\{z\} \times \operatorname{Sym}^{d-1}(\Sigma)\right)$.

Note that $n_{z}(u)$ only depends on the component of $\Sigma \backslash(\bigcup \boldsymbol{\alpha} \cup \bigcup \boldsymbol{\beta})$ in which $z$ lies and on the homotopy class of the Whitney disc $u$. Moreover, if the component of $z$ contains a boundary component of $\Sigma$ then $n_{z}(u)=0$. Indeed, we can choose $z$ on $\partial \Sigma$ and we can homotope $u$ to be disjoint from $\partial \operatorname{Sym}^{d}(\Sigma) \supset\{z\} \times \operatorname{Sym}^{d-1}(\Sigma)$, showing that $n_{z}(u)=0$. This last remark implies that we can run the Floer homology machinery without worrying about being in a manifold with boundary.

Definition 3.6 For a balanced diagram let $\mathcal{D}_{1}, \ldots, \mathcal{D}_{m}$ denote the closures of the components of $\Sigma \backslash(\bigcup \boldsymbol{\alpha} \cup \cup \boldsymbol{\beta})$ disjoint from $\partial \Sigma$. Then let $D(\Sigma, \boldsymbol{\alpha}, \boldsymbol{\beta})$ be the free abelian group generated by $\left\{\mathcal{D}_{1}, \ldots, \mathcal{D}_{m}\right\}$. This is of course isomorphic to $\mathbb{Z}^{m}$. We call an element of $D(\Sigma, \boldsymbol{\alpha}, \boldsymbol{\beta})$ a domain. An element $\mathcal{D}$ of $\mathbb{Z}_{\geq 0}^{m}$ is called a positive domain, we write $\mathcal{D} \geq 0$. A domain $\mathcal{P} \in D(\Sigma, \boldsymbol{\alpha}, \boldsymbol{\beta})$ is called a periodic domain if the boundary of the 2 -chain $\mathcal{P}$ is a sum of $\alpha$ - and $\beta$-curves.

Definition 3.7 For every $1 \leq i \leq m$ choose a point $z_{i} \in \mathcal{D}_{i}$. Then the domain of a Whitney disc $u$ is defined as

$$
\mathcal{D}(u)=\sum_{i=1}^{m} n_{z_{i}}(u) \mathcal{D}_{i} \in D(\Sigma, \boldsymbol{\alpha}, \boldsymbol{\beta}) .
$$

For $\phi \in \pi_{2}(\mathbf{x}, \mathbf{y})$ and $u$ a representative of the homotopy class $\phi$, let $\mathcal{D}(\phi)=\mathcal{D}(u)$.

Remark 3.8 If a Whitney disc $u$ is holomorphic then $\mathcal{D}(u) \geq 0$.

If $\mathbf{x} \in \mathbb{T}_{\alpha} \cap \mathbb{T}_{\beta}$ and if $u$ is a Whitney disc connecting $\mathbf{x}$ to $\mathbf{x}$ then $\mathcal{D}(u)$ is a periodic domain.

Definition 3.9 If $(\Sigma, \boldsymbol{\alpha}, \boldsymbol{\beta})$ is a balanced diagram defining the balanced sutured manifold $(M, \gamma)$ and if $\mathcal{P} \in D(\Sigma, \boldsymbol{\alpha}, \boldsymbol{\beta})$ is a periodic domain then we can naturally associate to $\mathcal{P}$ a homology class $H(\mathcal{P}) \in H_{2}(M ; \mathbb{Z})$ as follows. The boundary of the two-chain $\mathcal{P}$ is a sum $\sum_{i=1}^{d} a_{i} \alpha_{i}+\sum_{i=1}^{d} b_{i} \beta_{i}$. Let $A_{i}$ denote the core of the two-handle attached to $\alpha_{i}$ and $B_{i}$ the core of the two-handle attached to $\beta_{i}$. Then let

$$
H(\mathcal{P})=\left[\mathcal{P}+\sum_{i=1}^{d} a_{i} A_{i}+\sum_{i=1}^{d} b_{i} B_{i}\right] \in H_{2}(M ; \mathbb{Z}) .
$$

Lemma 3.10 If $H(\mathcal{P})=0$ then $\mathcal{P}=0$. 
Proof Since $\Sigma$ has no closed components we have that $H_{2}(\Sigma ; \mathbb{Z})=0$. Thus, if $\mathcal{P} \neq 0$ then $\partial \mathcal{P}=\sum_{i=1}^{d} a_{i} \alpha_{i}+\sum_{i=1}^{d} b_{i} \beta_{i} \neq 0$. Suppose for example that $a_{1} \neq 0$. This implies that $H(\mathcal{P})$ has nonzero algebraic intersection with the co-core $A_{1}^{\prime}$ of the two-handle attached to $\alpha_{1}$ (whose core is $\left.A_{1}\right)$. Since $\left[A_{1}^{\prime}\right] \neq 0$ in $H_{1}(M, \partial M ; \mathbb{Z}$ ) we get that $H(\mathcal{P}) \neq 0$.

Definition 3.11 A balanced diagram $(\Sigma, \boldsymbol{\alpha}, \boldsymbol{\beta})$ is called admissible if every periodic domain $\mathcal{P} \neq 0$ has both positive and negative coefficients.

Corollary 3.12 If $(M, \gamma)$ is a balanced sutured manifold such that $H_{2}(M ; \mathbb{Z})$ is 0 and if $(\Sigma, \boldsymbol{\alpha}, \boldsymbol{\beta})$ is an arbitrary balanced diagram defining $(M, \gamma)$ then there are no nonzero periodic domains in $D(\Sigma, \boldsymbol{\alpha}, \boldsymbol{\beta})$. Thus any balanced diagram defining $(M, \gamma)$ is automatically admissible.

Definition 3.13 Let $\mathbf{x}, \mathbf{y} \in \mathbb{T}_{\alpha} \cap \mathbb{T}_{\beta}$. A domain $\mathcal{D} \in D(\Sigma, \boldsymbol{\alpha}, \boldsymbol{\beta})$ is said to connect $\mathbf{x}$ to $\mathbf{y}$ if for every $1 \leq i \leq d$ the equalities $\partial\left(\alpha_{i} \cap \partial \mathcal{D}\right)=\left(\mathbf{x} \cap \alpha_{i}\right)-\left(\mathbf{y} \cap \alpha_{i}\right)$ and $\partial\left(\beta_{i} \cap \partial \mathcal{D}\right)=\left(\mathbf{x} \cap \beta_{i}\right)-\left(\mathbf{y} \cap \beta_{i}\right)$ hold. We will denote by $D(\mathbf{x}, \mathbf{y})$ the set of domains connecting $\mathbf{x}$ to $\mathbf{y}$.

Note that if $\phi \in \pi_{2}(\mathbf{x}, \mathbf{y})$ then $\mathcal{D}(\phi) \in D(\mathbf{x}, \mathbf{y})$.

Lemma 3.14 If the balanced diagram $(\Sigma, \boldsymbol{\alpha}, \boldsymbol{\beta})$ is admissible then for every pair $\mathbf{x}, \mathbf{y} \in \mathbb{T}_{\alpha} \cap \mathbb{T}_{\beta}$ the set $\{\mathcal{D} \in D(\mathbf{x}, \mathbf{y}): \mathcal{D} \geq 0\}$ is finite.

Proof The argument that follows can be found in the proof of [9, Lemma 4.13]. If $D(\mathbf{x}, \mathbf{y}) \neq \varnothing$ then fix an element $\mathcal{D}_{0} \in D(\mathbf{x}, \mathbf{y})$. Then every element $\mathcal{D} \in D(\mathbf{x}, \mathbf{y})$ can be written as $\mathcal{D}=\mathcal{D}_{0}+\mathcal{P}$, where $\mathcal{P} \in D(\mathbf{x}, \mathbf{x})$ is a periodic domain. Hence if $\mathcal{D} \geq 0$ then $\mathcal{P} \geq-\mathcal{D}_{0}$.

So the lemma follows if we show that the set $Q=\left\{\mathcal{P} \in D(\mathbf{x}, \mathbf{x}): \mathcal{P} \geq-\mathcal{D}_{0}\right\}$ is finite. We can think of $Q$ as a subset of the lattice $\mathbb{Z}^{m} \subset \mathbb{R}^{m}$. If $Q$ had infinitely many elements, then we could find a sequence $\left(p_{j}\right)_{j=1}^{\infty}$ in $Q$ with $\left\|p_{j}\right\| \rightarrow \infty$. Taking a subsequence we can suppose that $\left(p_{j} /\left\|p_{j}\right\|\right)$ converges to a unit vector $p$ in the vector space of periodic domains with real coefficients. Since the coefficients of $p_{j}$ are bounded below and $\left\|p_{j}\right\| \rightarrow \infty$ we get that $p \geq 0$. Thus the polytope consisting of vectors corresponding to real periodic domains with $\geq 0$ multiplicities also has a nonzero rational vector. After clearing denominators we obtain a nonzero integer periodic domain with nonnegative multiplicities. This contradicts the hypothesis of admissibility. 

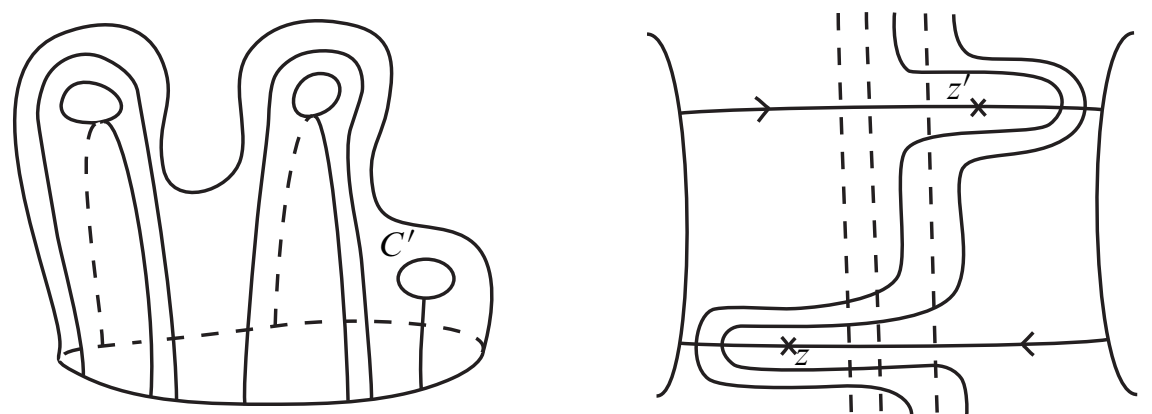

C

Figure 1: The picture on the left shows curves that represent a basis of $H_{1}(\Sigma, \partial \Sigma ; \mathbb{Z})$. On the right we can see the procedure to achieve admissibility. The dotted lines represent the $\alpha$ curves, and the solid lines represent the $\beta$ curves.

Proposition 3.15 Every balanced diagram $(\Sigma, \boldsymbol{\alpha}, \boldsymbol{\beta})$ is isotopic to an admissible one.

Proof Fix a boundary component $C \subset \partial \Sigma$. We can choose a set of pairwise disjoint, oriented and properly embedded arcs $\gamma_{1}, \ldots, \gamma_{l} \subset \Sigma$ such that for every $1 \leq i \leq l$ the endpoints $\partial \gamma_{i}$ lie in $\partial \Sigma$; furthermore these arcs generate the relative homology group $H_{1}(\Sigma, \partial \Sigma ; \mathbb{Z})$. This can be done as follows (see the left hand side of Figure 1). Let $\Sigma^{\prime}$ denote the surface obtained from $\Sigma$ by gluing a disc to every component of $\partial \Sigma \backslash C$. Let $g$ denote the genus of $\Sigma^{\prime}$. Then we can choose a set of $2 g$ curves in $\Sigma^{\prime}$ as above, that are also disjoint from $\partial \Sigma \backslash C$. Finally, for each component $C^{\prime}$ of $\partial \Sigma \backslash C$ connect $C$ and $C^{\prime}$ with a $\gamma$ curve. Note that $\Sigma \backslash\left(\partial \Sigma \cup \bigcup_{i=1}^{l} \gamma_{i}\right)$ is homeomorphic to an open disc.

We perform an isotopy of the $\beta$ curves in a regular neighborhood of $\gamma_{1} \cup \cdots \cup \gamma_{l}$ as described in the proof of [7, Proposition 3.6]. Specifically, for every $1 \leq i \leq l$ choose an oppositely oriented parallel copy $\gamma_{i}^{\prime}$ of $\gamma_{i}$. Using a finger move isotope the $\beta$ curves intersecting $\gamma_{i}$ towards the endpoint of $\gamma_{i}$ so that there is a point $z_{i} \in \gamma_{i}$ separating $\boldsymbol{\alpha} \cap \gamma_{i}$ from $\boldsymbol{\beta} \cap \gamma_{i}$. Perform a similar isotopy of the $\beta$ curves in a neighborhood of each $\gamma_{i}^{\prime}$. The point $z_{i}^{\prime} \in \gamma_{i}^{\prime}$ separates $\boldsymbol{\alpha} \cap \gamma_{i}^{\prime}$ from $\boldsymbol{\beta} \cap \gamma_{i}^{\prime}$. See the right-hand side of Figure 1.

We claim the diagram obtained this way is admissible. Let $\mathcal{P}$ be a periodic domain. Then

$$
\partial \mathcal{P}=\sum_{i=1}^{d} a_{i} \cdot \alpha_{i}+\sum_{i=1}^{d} b_{i} \cdot \beta_{i}=A+B
$$

Algebraic 83 Geometric Topology, Volume 6 (2006) 
First suppose that there is an $1 \leq i \leq l$ such that the algebraic intersection $A \cap \gamma_{i} \neq 0$. Since the multiplicity of $\mathcal{P}$ at the points of $\partial \Sigma$ is 0 we get that the multiplicity of $\mathcal{P}$ at $z_{i}$ is $A \cap \gamma_{i}$ and at $z_{i}^{\prime}$ it is $A \cap \gamma_{i}^{\prime}=-A \cap \gamma_{i}$ (see the right-hand side of Figure 1). Indeed, $z_{i}$ separates $A \cap \gamma_{i}$ from $B \cap \gamma_{i}$ on $\gamma_{i}$ and $z_{i}^{\prime}$ separates $A \cap \gamma_{i}^{\prime}$ from $B \cap \gamma_{i}^{\prime}$ on $\gamma_{i}^{\prime}$. Thus $\mathcal{P}$ has both positive and negative multiplicities.

On the other hand, if for every $1 \leq i \leq l$ the intersection number $A \cap \gamma_{i}=0$, then since $\gamma_{1}, \ldots, \gamma_{l}$ span $H_{1}(\Sigma, \partial \Sigma ; \mathbb{Z})$, we get that $A$ is null-homologous in $\Sigma$. Indeed, in this case $A$ is homologous to a curve lying in $\Sigma \backslash\left(\gamma_{1} \cup \cdots \cup \gamma_{l}\right) \approx D^{2}$. Since the elements of $\boldsymbol{\alpha}$ are linearly independent is $H_{1}(\Sigma ; \mathbb{Z})$ we get that for every $1 \leq j \leq d$ the coefficient $a_{j}=0$. But $\partial \mathcal{P}=A+B$ implies that $B$ is homologous with $-A$ in $\Sigma$, thus $B \sim 0$. So we get that $b_{j}=0$ for every $1 \leq j \leq d$. Thus in this case $\mathcal{P}=0$.

\section{Spin $^{c}$ structures}

In this section $(M, \gamma)$ denotes a connected balanced sutured manifold.

Notation 4.1 Let $v_{0}$ be the nonzero vector field along $\partial M$ that points into $M$ along $R_{-}(\gamma)$, points out of $M$ along $R_{+}(\gamma)$, and on $\gamma$ it is the gradient of the height function $s(\gamma) \times I \rightarrow I$. The space of such vector fields is contractible.

The field $v_{0}^{\perp}$ is an oriented two-plane field along $\partial M$. We will use the notation

$$
\delta=c_{1}\left(v_{0}^{\perp}\right)=e\left(v_{0}^{\perp}\right) \in H^{2}(\partial M ; \mathbb{Z}) .
$$

Definition 4.2 Let $v$ and $w$ be vector fields on $M$ that agree with $v_{0}$ on $\partial M$. We say that $v$ and $w$ are homologous if there is an open ball $B \subset \operatorname{Int}(M)$ such that $v \mid(M \backslash B)$ is homotopic to $w \mid(M \backslash B)$ rel $\partial M$. We define $\operatorname{Spin}^{c}(M, \gamma)$ to be the set of homology classes of nonzero vector fields $v$ on $M$ such that $v \mid \partial M=v_{0}$.

Remark 4.3 Let $f$ be a Morse function as in Proposition 2.13. Then the vector field $\operatorname{grad}(f) \mid \partial M=v_{0}$, the number $d$ of index 1 and 2 critical points of $f$ agree, and $f$ has no index 0 or 3 critical points. Choose $d$ pairwise disjoint balls in $M$, each containing exactly one index 1 and one index 2 critical point of $f$. Then we can modify $\operatorname{grad}(f)$ on these balls so that we obtain a nonzero vector field on $M$ such that $v \mid \partial M=v_{0}$. This shows that $\operatorname{Spin}^{c}(M, \gamma) \neq \varnothing$. From obstruction theory we get that $\operatorname{Spin}^{c}(M, \gamma)$ is an affine space over $H^{2}(M, \partial M ; \mathbb{Z})$.

Next we define the Chern class of a $\operatorname{Spin}^{c}$ structure. Let $i: \partial M \rightarrow M$ denote the embedding. If $v$ is the vector field constructed in Remark 4.3 then using the naturality of Chern classes we see that $i^{*}\left(c_{1}\left(v^{\perp}\right)\right)=\delta$, thus $\delta \in \operatorname{Im}\left(i^{*}\right)$. 
Definition 4.4 For $\mathfrak{s} \in \operatorname{Spin}^{c}(M, \gamma)$ defined by a vector field $v$ on $M$, let the class $c_{1}(\mathfrak{s})=c_{1}\left(v^{\perp}\right) \in\left(i^{*}\right)^{-1}(\delta)$ where $v^{\perp}$ is the oriented two-plane field on $M$ perpendicular to $v$. Note that a priori we only know that $c_{1}\left(v^{\perp}\right) \in H^{2}(M ; \mathbb{Z})$, but since $v \mid \partial M=v_{0}$ we get that $c_{1}\left(v^{\perp}\right) \in\left(i^{*}\right)^{-1}(\delta)$.

Fix a balanced diagram $(\Sigma, \boldsymbol{\alpha}, \boldsymbol{\beta})$ for $(M, \gamma)$.

Definition 4.5 To each $\mathbf{x} \in \mathbb{T}_{\alpha} \cap \mathbb{T}_{\beta}$ we assign a $\operatorname{Spin}^{c}$ structure $\mathfrak{s}(\mathbf{x}) \in \operatorname{Spin}^{c}(M, \gamma)$ as follows. Choose a Morse function $f$ on $M$ compatible with the given balanced diagram $(\Sigma, \boldsymbol{\alpha}, \boldsymbol{\beta})$. Then $\mathbf{x}$ corresponds to a multi-trajectory $\gamma_{\mathbf{x}}$ of $\operatorname{grad}(f)$ connecting the index one and two critical points of $f$. In a regular neighborhood $N\left(\gamma_{\mathbf{x}}\right)$ we can modify $\operatorname{grad}(f)$ to obtain a nonzero vector field $v$ on $M$ such that $v \mid \partial M=v_{0}$. We define $\mathfrak{s}(\mathbf{x})$ to be the homology class of this vector field $v$.

Definition 4.6 Let $\mathbf{x}, \mathbf{y} \in \mathbb{T}_{\alpha} \cap \mathbb{\mathbb { T }}_{\beta}$ and let $\gamma_{\mathbf{x}}, \gamma_{\mathbf{y}}$ be the corresponding multi-trajectories, thought of as one-chains in $M$. Then define $\epsilon(x, y)=\gamma_{\mathbf{x}}-\gamma_{\mathbf{y}} \in H_{1}(M ; \mathbb{Z})$.

Alternatively, we can define $\epsilon(\mathbf{x}, \mathbf{y})$ in the following manner. Choose paths $a: I \rightarrow \mathbb{T}_{\alpha}$ and $b: I \rightarrow \mathbb{T}_{\beta}$ with $\partial a=\partial b=\mathbf{x}-\mathbf{y}$. Then $a-b$ can be viewed as a one-cycle in $\Sigma$ whose homology class in $M$ is $\epsilon(\mathbf{x}, \mathbf{y})$. This is independent of the choice of $a$ and $b$.

Lemma 4.7 For $\mathbf{x}, \mathbf{y} \in \mathbb{T}_{\alpha} \cap \mathbb{T}_{\beta}$ we have that $\mathfrak{s}(\mathbf{x})-\mathfrak{s}(\mathbf{y})=P D[\epsilon(\mathbf{x}, \mathbf{y})]$, where $P D: H_{1}(M, \mathbb{Z}) \rightarrow H^{2}(M, \partial M ; \mathbb{Z})$ is the Poincaré duality map.

Proof The vector fields $\mathfrak{s}(\mathbf{x})$ and $\mathfrak{s}(\mathbf{y})$ differ only in a neighborhood of $\gamma_{\mathbf{x}}-\gamma_{\mathbf{y}}$. It is now a local calculation to see that $\mathfrak{s}(\mathbf{x})-\mathfrak{s}(\mathbf{y})=P D\left[\gamma_{\mathbf{x}}-\gamma_{\mathbf{y}}\right]$ (see [9, Lemma 2.19]).

Corollary 4.8 If $\mathfrak{s}(\mathbf{x}) \neq \mathfrak{s}(\mathbf{y})$ then $D(\mathbf{x}, \mathbf{y})=\varnothing$. In particular, there is no Whitney disc connecting $\mathbf{x}$ and $\mathbf{y}$. If $\pi_{1}\left(\operatorname{Sym}^{d}(\Sigma)\right)=H_{1}\left(\operatorname{Sym}^{d}(\Sigma) ; \mathbb{Z}\right)$ then the converse also holds, ie, $\mathfrak{s}(\mathbf{x})=\mathfrak{s}(\mathbf{y})$ implies that $\pi_{2}(\mathbf{x}, \mathbf{y}) \neq \varnothing$.

Proposition 4.9 If $d>1$ then $\pi_{1}\left(\operatorname{Sym}^{d}(\Sigma)\right)=H_{1}\left(\operatorname{Sym}^{d}(\Sigma) ; \mathbb{Z}\right)$.

Proof The proof is analogous to [9, Lemma 2.6]. Let $\gamma: S^{1} \rightarrow \operatorname{Sym}^{d}(\Sigma)$ be a nullhomologous curve that misses the diagonal. Then there is a 2 -manifold with boundary $F$, a map $i: F \rightarrow \Sigma$ and a $d$-fold covering $\pi: \partial F \rightarrow S^{1}$ such that $(i \mid \partial F) \circ \pi^{-1}=\gamma$. By increasing the genus of $F$ is necessary, we can extend the $d$-fold covering of $S^{1}$ to a branched $d$-fold covering $\pi: F \rightarrow D^{2}$. Then the map $i \circ \pi^{-1}: D^{2} \rightarrow \operatorname{Sym}^{d}(\Sigma)$ shows that $\gamma$ is null-homotopic. 


\section{Maslov index}

Fix a balanced sutured manifold $(M, \gamma)$ and a balanced diagram $(\Sigma, \boldsymbol{\alpha}, \boldsymbol{\beta})$ defining it.

Notation 5.1 For $\mathbf{x}, \mathbf{y} \in \mathbb{T}_{\alpha} \cap \mathbb{T}_{\beta}$ and for a homotopy class $\phi \in \pi_{2}(\mathbf{x}, \mathbf{y})$ let $\mathcal{M}(\phi)$ denote the moduli space of pseudo-holomorphic representatives of $\phi$, and let $\widehat{\mathcal{M}}(\phi)$ be the quotient of this moduli space by the action of $\mathbb{R}$. Let $\mu(\phi)$ denote the Maslov index of $\phi$, ie, the expected dimension of $\mathcal{M}(\phi)$.

Theorem 5.2 For $\mathbf{x} \in \mathbb{T}_{\alpha} \cap \mathbb{T}_{\beta}$ and $\psi \in \pi_{2}(\mathbf{x}, \mathbf{x})$ we have

$$
\mu(\psi)=\left\langle c_{1}(\mathfrak{s}(\mathbf{x})), H(\mathcal{D}(\psi))\right\rangle .
$$

Proof See [9, Theorem 4.9].

Corollary 5.3 Suppose that for $\phi_{1}, \phi_{2} \in \pi_{2}(\mathbf{x}, \mathbf{y})$ we have that $\mathcal{D}\left(\phi_{1}\right)=\mathcal{D}\left(\phi_{2}\right)$. Then $\mu\left(\phi_{1}\right)=\mu\left(\phi_{2}\right)$.

Proof The homotopy class $\psi=\phi_{1} \bar{\phi}_{2} \in \pi_{2}(\mathbf{x}, \mathbf{x})$ satisfies $\mathcal{D}(\psi)=\mathcal{D}\left(\phi_{1}\right)-\mathcal{D}\left(\phi_{2}\right)=0$ and $\mu(\psi)=\mu\left(\phi_{1}\right)-\mu\left(\phi_{2}\right)$. The result then follows from Theorem 5.2 using the fact that $H(\mathcal{D}(\psi))=0$.

This justifies the following definition.

Definition 5.4 We define the Maslov index of a domain $\mathcal{D} \in D(\Sigma, \boldsymbol{\alpha}, \boldsymbol{\beta})$ as follows. If there exists a homotopy class $\phi$ of Whitney discs such that $\mathcal{D}(\phi)=\mathcal{D}$ then define $\mu(\mathcal{D}):=\mu(\phi)$. Otherwise we define $\mu(\mathcal{D})$ to be $-\infty$. Furthermore, let $\mathcal{M}(\mathcal{D})$ denote the moduli space of holomorphic Whitney discs $u$ such that $\mathcal{D}(u)=\mathcal{D}$ and let $\widehat{\mathcal{M}}(\mathcal{D})=\mathcal{M}(\mathcal{D}) / \mathbb{R}$.

Thus we can rephrase Theorem 5.2 as follows.

Theorem 5.5 For $\mathbf{x} \in \mathbb{T}_{\alpha} \cap \mathbb{T}_{\beta}$ and $\mathcal{P} \in D(\mathbf{x}, \mathbf{x})$ such that $\mu(\mathcal{P}) \neq-\infty$ we have

$$
\mu(\mathcal{P})=\left\langle c_{1}(\mathfrak{s}(\mathbf{x})), H(\mathcal{P})\right\rangle .
$$




\section{Energy bounds}

First we recall the definition of the energy of a map of a planar domain into a Riemannian manifold.

Definition 6.1 Let $\Omega$ be a domain in $\mathbb{C}$ and let $(X, g)$ be a Riemannian manifold. The energy of a smooth map $u: \Omega \rightarrow X$ is given by

$$
E(u)=\frac{1}{2} \int_{\Omega}|d u|^{2} .
$$

Let $\eta$ be a Kähler form on $\Sigma$.

Definition 6.2 The area of a domain $\mathcal{D}=\sum_{i=1}^{m} n_{i} \mathcal{D}_{i} \in D(\Sigma, \boldsymbol{\alpha}, \boldsymbol{\beta})$ is defined as

$$
\mathcal{A}(\mathcal{D})=\sum_{i=1}^{m} n_{i} \cdot \operatorname{Area}_{\eta}\left(\mathcal{D}_{i}\right),
$$

where $\operatorname{Area}_{\eta}\left(\mathcal{D}_{i}\right)=\int_{\mathcal{D}_{i}} \eta$.

Theorem 6.3 There is a constant $C$ which depends only on the balanced diagram $(\Sigma, \boldsymbol{\alpha}, \boldsymbol{\beta})$ and $\eta$ such that for any smooth Whitney disc

$$
u:(\mathbb{D}, \partial \mathbb{D}) \rightarrow\left(\operatorname{Sym}^{d}(\Sigma), \mathbb{T}_{\alpha} \cup \mathbb{T}_{\beta}\right)
$$

we have the energy bound

$$
E(u) \leq C \cdot \mathcal{A}(\mathcal{D}(u))
$$

Proof The proof is analogous to the proof of [9, Lemma 3.5]. We use the fact that $\Sigma$ is compact. See also the paragraph below [9, Remark 3.7].

Corollary 6.4 For any $\mathcal{D} \in D(\Sigma, \boldsymbol{\alpha}, \boldsymbol{\beta})$ such that $\mu(\mathcal{D})=1$ the moduli space $\widehat{\mathcal{M}}(\mathcal{D})$ is a compact zero-dimensional manifold.

Proof This follows from Theorem 6.3 using Gromov compactness.

Lemma 6.5 Every pseudo-holomorphic map

$$
u:(\mathbb{D}, \partial \mathbb{D}) \rightarrow\left(\operatorname{Sym}^{d}(\Sigma), \mathbb{T}_{\alpha}\right)
$$

is constant. The same holds for pseudo-holomorphic maps

$$
u^{\prime}:(\mathbb{D}, \partial \mathbb{D}) \rightarrow\left(\operatorname{Sym}^{d}(\Sigma), \mathbb{T}_{\beta}\right) .
$$

Finally, every pseudo-holomorphic sphere $v: S^{2} \rightarrow \operatorname{Sym}^{d}(\Sigma)$ is constant. 
Proof The boundary of the domain $\mathcal{D}(u)$ is a linear combination of the $\alpha$ curves. Since $\alpha_{1}, \ldots, \alpha_{d}$ are linearly independent in $\Sigma$, this implies that $\mathcal{D}(u)=0$. Thus using Theorem 6.3 we get that $E(u) \leq C \cdot \mathcal{A}(0)=0$, so $E(u)=0$. A pseudo-holomorphic map with zero energy is constant. A similar argument applies to $u^{\prime}$.

The domain of $v$ is a 2 -cycle. But $\Sigma$ has no closed components, so $\mathcal{D}(v)=0$. The fact that $v$ is constant now follows similarly.

\section{Definition of the chain complex}

Let $(M, \gamma)$ be a balanced sutured manifold and $(\Sigma, \boldsymbol{\alpha}, \boldsymbol{\beta})$ an admissible balanced diagram defining it. Fix a coherent system of orientations as in [9, Definition 3.11].

Definition 7.1 Let $C F(\Sigma, \alpha, \beta)$ be the free abelian group generated by the points in $\mathbb{T}_{\alpha} \cap \mathbb{T}_{\beta}$. We define an endomorphism $\partial: C F(\Sigma, \boldsymbol{\alpha}, \boldsymbol{\beta}) \rightarrow C F(\Sigma, \boldsymbol{\alpha}, \boldsymbol{\beta})$ so that for each generator $\mathbf{x} \in \mathbb{T}_{\alpha} \cap \mathbb{\mathbb { T }}_{\beta}$ we have

$$
\partial \mathbf{x}=\sum_{\mathbf{y} \in \mathbb{T}_{\alpha} \cap \mathbb{T}_{\beta}} \sum_{\{\mathcal{D} \in D(\mathbf{x}, \mathbf{y}): \mu(\mathcal{D})=1\}} \# \widehat{\mathcal{M}}(\mathcal{D}) \cdot \mathbf{y} .
$$

Since the diagram is admissible Lemma 3.14 ensures that $D(\mathbf{x}, \mathbf{y})$ has only finitely many positive elements. But we know that from $\mathcal{M}(\mathcal{D}) \neq \varnothing$ it follows that $\mathcal{D} \geq 0$. Corollary 6.4 implies that if $\mu(\mathcal{D})=1$ then $\widehat{\mathcal{M}}(\mathcal{D})$ is a compact zero-dimensional manifold, and the coherent orientation system makes it oriented. Thus $\# \widehat{\mathcal{M}}(\mathcal{D})$ makes sense, and the sum above has only finitely many nonzero terms.

Theorem 7.2 The pair $(C F(\Sigma, \boldsymbol{\alpha}, \boldsymbol{\beta}), \partial)$ is a chain complex, ie, $\partial^{2}=0$.

Proof $\partial^{2}=0$ follows as in [9, Theorem 4.1]. Boundary degenerations and spheres bubbling off are excluded by Lemma 6.5 .

Definition 7.3 For $\mathfrak{s} \in \operatorname{Spin}^{c}(M, \gamma)$ let $C(\Sigma, \boldsymbol{\alpha}, \boldsymbol{\beta}, \mathfrak{s})$ be the free abelian group generated by those intersection points $\mathbf{x} \in \mathbb{T}_{\alpha} \cap \mathbb{T}_{\beta}$ for which $\mathfrak{s}(\mathbf{x})=\mathfrak{s}$.

It follows from Corollary 4.8 that $C(\Sigma, \boldsymbol{\alpha}, \boldsymbol{\beta}, \mathfrak{s})$ is a subcomplex of $C(\Sigma, \boldsymbol{\alpha}, \boldsymbol{\beta})$ and

$$
(C(\Sigma, \boldsymbol{\alpha}, \boldsymbol{\beta}), \partial)=\bigoplus_{\mathfrak{s} \in \operatorname{Spin}^{c}(M, \gamma)}(C(\Sigma, \boldsymbol{\alpha}, \boldsymbol{\beta}, \mathfrak{s}), \partial) .
$$


Definition 7.4 We define $\operatorname{SFH}(\Sigma, \boldsymbol{\alpha}, \boldsymbol{\beta})$ to be the homology of the chain complex $(C F(\Sigma, \boldsymbol{\alpha}, \boldsymbol{\beta}), \partial)$. Similarly, for $\mathfrak{s} \in \operatorname{Spin}^{c}(M, \gamma)$ let $\operatorname{SFH}(\Sigma, \boldsymbol{\alpha}, \boldsymbol{\beta}, \mathfrak{s})$ be the homology of $(C F(\Sigma, \boldsymbol{\alpha}, \boldsymbol{\beta}, \mathfrak{s}), \partial)$.

Theorem 7.5 If the admissible balanced diagrams $(\Sigma, \boldsymbol{\alpha}, \boldsymbol{\beta})$ and $\left(\Sigma^{\prime}, \boldsymbol{\alpha}^{\prime}, \boldsymbol{\beta}^{\prime}\right)$ define the same sutured manifold then

and

$$
\operatorname{SFH}(\Sigma, \boldsymbol{\alpha}, \boldsymbol{\beta})=\operatorname{SFH}\left(\Sigma^{\prime}, \boldsymbol{\alpha}^{\prime}, \boldsymbol{\beta}^{\prime}\right)
$$

holds for every $\mathfrak{s} \in \operatorname{Spin}^{c}(M, \gamma)$.

Proof This follows from Proposition 2.15 as in [9].

Thus we can make the following definition.

Definition 7.6 For $(M, \gamma)$ a balanced sutured manifold, we define the sutured Floer homology $\operatorname{SFH}(M, \gamma)$ as follows. Choose an admissible balanced diagram $(\Sigma, \boldsymbol{\alpha}, \boldsymbol{\beta})$ defining $(M, \gamma)$. Then let $\operatorname{SFH}(M, \gamma)=\operatorname{SFH}(\Sigma, \boldsymbol{\alpha}, \boldsymbol{\beta})$. For $\mathfrak{s} \in \operatorname{Spin}^{c}(M, \gamma)$ define $\operatorname{SFH}(M, \gamma, \mathfrak{s})$ to be $\operatorname{SFH}(\Sigma, \boldsymbol{\alpha}, \boldsymbol{\beta}, \mathfrak{s})$.

\section{Relative gradings}

Suppose that $d>1$ in the balanced diagram $(\Sigma, \boldsymbol{\alpha}, \boldsymbol{\beta})$. Then, using Proposition 4.9 we get that $\pi_{1}\left(\operatorname{Sym}^{d}(\Sigma)\right)=H_{1}\left(\operatorname{Sym}^{d}(\Sigma) ; \mathbb{Z}\right)$. Thus, according to Corollary 4.8 , for every $\mathbf{x}, \mathbf{y} \in \mathbb{T}_{\alpha} \cap \mathbb{T}_{\beta}$ the equality $\mathfrak{s}(\mathbf{x})=\mathfrak{s}(\mathbf{y})$ implies that $\pi_{2}(\mathbf{x}, \mathbf{y}) \neq \varnothing$. Note that every balanced sutured manifold $(M, \gamma)$ has a diagram with $d>1$, we can achieve this by stabilizing an arbitrary balanced diagram defining $(M, \gamma)$.

Definition 8.1 For $\mathfrak{s} \in \operatorname{Spin}^{c}(M, \gamma)$ let

$$
\mathfrak{d}(\mathfrak{s})=\underset{\xi \in H_{2}(M ; \mathbb{Z})}{\operatorname{gcd}}\left\langle c_{1}(\mathfrak{s}), \xi\right\rangle .
$$

Definition 8.2 Let $\mathfrak{s} \in \operatorname{Spin}^{c}(M, \gamma)$ and let $(\Sigma, \boldsymbol{\alpha}, \boldsymbol{\beta})$ be an admissible balanced diagram with $d>1$ defining $(M, \gamma)$. Then we define a relative $\mathbb{Z}_{\mathfrak{o}(\mathfrak{s})}$ grading on $C F(\Sigma, \boldsymbol{\alpha}, \boldsymbol{\beta}, \mathfrak{s})$ such that for any $\mathbf{x}, \mathbf{y} \in \mathbb{T}_{\alpha} \cap \mathbb{T}_{\beta}$ with $\mathfrak{s}(\mathbf{x})=\mathfrak{s}(\mathbf{y})=\mathfrak{s}$ we have

$$
\operatorname{gr}(\mathbf{x}, \mathbf{y})=\mu(\phi) \quad \bmod \mathfrak{d}(\mathfrak{s}),
$$

where $\phi \in \pi_{2}(\mathbf{x}, \mathbf{y})$ is an arbitrary homotopy class. 
The number $\operatorname{gr}(\mathbf{x}, \mathbf{y})$ is independent of the choice of $\phi$ because of Theorem 5.5. From the definition of $\partial$ it is clear that gr descends to a relative grading on $\operatorname{SFH}(M, \gamma, \mathfrak{s})$. This grading is independent of the balanced diagram defining the sutured manifold $(M, \gamma)$.

\section{Special cases and sample computations}

Proposition 9.1 Let $Y$ be a closed connected oriented 3-manifold. Then

$$
\widehat{\mathrm{HF}}(Y) \approx \mathrm{SFH}(Y(1)) \text {. }
$$

Recall that $Y(1)$ was introduced in Example 2.3. For the definition of $\widehat{\mathrm{HF}}(Y)$ see [9].

Proof Let $(\Sigma, \boldsymbol{\alpha}, \boldsymbol{\beta}, z)$ be a weakly admissible Heegaard diagram defining $Y$. Choose a small neighborhood $U$ of $z$ diffeomorphic to an open disc and let $\Sigma^{\prime}=\Sigma \backslash U$. Then $\left(\Sigma^{\prime}, \boldsymbol{\alpha}, \boldsymbol{\beta}\right)$ is an admissible balanced sutured diagram defining $Y(1)$. Since each $\mathcal{D} \in D\left(\Sigma^{\prime}, \boldsymbol{\alpha}, \boldsymbol{\beta}\right)$ has multiplicity zero at $\partial \Sigma^{\prime}$ the chain complexes $\widehat{C F}(\Sigma, \boldsymbol{\alpha}, \boldsymbol{\beta}, z)$ and $C F\left(\Sigma^{\prime}, \boldsymbol{\alpha}, \boldsymbol{\beta}\right)$ are isomorphic.

In Example 2.4 for every link $L$ in a closed connected oriented 3 -manifold $Y$ we defined a balanced sutured manifold $Y(L)$. In [7] an invariant $\widehat{\mathrm{HFL}}(\vec{L})$ was defined for oriented links $\vec{L} \subset Y$. Suppose that $L$ has $l$ components, then $\widehat{\operatorname{HFL}}(\vec{L})$ is computed using $2 l$-pointed Heegaard diagrams and Floer homology is taken with coefficients in $\mathbb{Z}_{2}$.

Proposition 9.2 If $Y$ is a closed connected oriented 3-manifold, $L \subset Y$ is a link, and $\vec{L}$ is an arbitrary orientation of $L$ then

$$
\widehat{\operatorname{HFL}}(\vec{L}) \approx \operatorname{SFH}(Y(L)) \otimes \mathbb{Z}_{2} .
$$

If $L$ has only one component, ie, if $L$ is a knot $K$, then

$$
\widehat{\operatorname{HFK}}(Y, K) \approx \operatorname{SFH}(Y(K)) \text {. }
$$

For the definition of $\widehat{\mathrm{HFK}}$ see [8] or [12].

Proof Let $l$ be the number of components of the link $L$. If $(\Sigma, \boldsymbol{\alpha}, \boldsymbol{\beta}, \mathbf{w}, \mathbf{z})$ is a weakly admissible $2 l$-pointed balanced Heegaard diagram of $\vec{L}$ in the sense of [7] then remove an open regular neighborhood of $\mathbf{w} \cup \mathbf{z}$ to obtain a compact surface $\Sigma^{\prime}$. The diagram $\left(\Sigma^{\prime}, \boldsymbol{\alpha}, \boldsymbol{\beta}\right)$ is a balanced diagram defining the sutured manifold $Y(L)$. It is now clear that the two chain complexes are isomorphic.

Algebraic $8 \mathcal{G}$ Geometric Topology, Volume 6 (2006) 
Remark 9.3 Suppose that $(M, \gamma)$ is a balanced sutured manifold such that $\partial M$ is a torus and $s(\gamma)$ consists of two components that represent the same homology class in $H_{1}(\partial M ; \mathbb{Z})$. Then $\operatorname{SFH}(M, \gamma)$ can be computed as the knot Floer homology of the knot obtained from $(M, \gamma)$ using the Dehn filling construction described in Example 2.4 .

Proposition 9.4 If $(M, \gamma)$ is a product sutured manifold then

$$
\operatorname{SFH}(M, \gamma) \approx \mathbb{Z}
$$

Proof Since $(M, \gamma)$ is product there is a compact oriented surface $R$ with no closed components such that $(M, \gamma)=(R \times I, \partial R \times I)$. Then $(R, \boldsymbol{\alpha}, \boldsymbol{\beta})$ is a balanced diagram defining $(M, \gamma)$, where $\boldsymbol{\alpha}=\varnothing$ and $\boldsymbol{\beta}=\varnothing$. Since $H_{2}(M ; \mathbb{Z})=0$ any balanced diagram defining $(M, \gamma)$ is admissible. Thus $\operatorname{Sym}^{0}(\Sigma)=\{\mathrm{pt}\}$ and $\mathbb{T}_{\alpha}=\{\mathrm{pt}\}=\mathbb{T}_{\beta}$. Hence $\mathbb{T}_{\alpha} \cap \mathbb{T}_{\beta}$ consists of a single point. Alternatively, we can stabilize the above diagram and obtain the same result.

Remark 9.5 Let $P$ denote the Poincaré 3 -sphere. Then the balanced sutured manifold $P(1)$ is not a product. On the other hand $\operatorname{SFH}(P(1)) \approx \widehat{\mathrm{HF}}(P) \approx \mathbb{Z}$ by Proposition 9.1 .

Definition 9.6 A sutured manifold $(M, \gamma)$ is called irreducible if every 2-sphere smoothly embedded in $M$ bounds a 3-ball.

Question 9.7 Is the converse of Proposition 9.4 true under certain hypotheses? More precisely, suppose that the manifold $(M, \gamma)$ is irreducible and $H_{2}(M ; \mathbb{Z})=0$. Then does $\operatorname{SFH}(M, \gamma)=\mathbb{Z}$ imply that $(M, \gamma)$ is a product sutured manifold?

Next we recall the definition of a sutured manifold decomposition; see [1, Definition 3.1].

Definition 9.8 Let $(M, \gamma)$ be a sutured manifold and $S$ a properly embedded oriented surface in $M$ such that for every component $\lambda$ of $S \cap \gamma$, one of (1)-(3) holds:

(1) $\lambda$ is a properly embedded nonseparating arc in $\gamma$.

(2) $\lambda$ is a simple closed curve in an annular component $A$ of $\gamma$ in the same homology class as $A \cap s(\gamma)$.

(3) $\lambda$ is a homotopically nontrivial curve in a torus component $T$ of $\gamma$, and if $\delta$ is another component of $T \cap S$, then $\lambda$ and $\delta$ represent the same homology class in $H_{1}(T)$. 
Then $S$ defines a sutured manifold decomposition

$$
(M, \gamma) \rightsquigarrow{ }^{S}\left(M^{\prime}, \gamma^{\prime}\right),
$$

where $M^{\prime}=M \backslash \operatorname{Int}(N(S))$ and

$$
\gamma^{\prime}=\left(\gamma \cap M^{\prime}\right) \cup N\left(S_{+}^{\prime} \cap R_{-}(\gamma)\right) \cup N\left(S_{-}^{\prime} \cap R_{+}(\gamma)\right) .
$$

Also,

$$
R_{+}\left(\gamma^{\prime}\right)=\left(\left(R_{+}(\gamma) \cap M^{\prime}\right) \cup S_{+}^{\prime}\right) \backslash \operatorname{Int}\left(\gamma^{\prime}\right)
$$

and

$$
R_{-}\left(\gamma^{\prime}\right)=\left(\left(R_{-}(\gamma) \cap M^{\prime}\right) \cup S_{-}^{\prime}\right) \backslash \operatorname{Int}\left(\gamma^{\prime}\right),
$$

where $S_{+}^{\prime}\left(S_{-}^{\prime}\right)$ is the component of $\partial N(S) \cap M^{\prime}$ whose normal vector points out of (into) $M^{\prime}$.

Remark 9.9 In other words the sutured manifold $\left(M^{\prime}, \gamma^{\prime}\right)$ is constructed by splitting $M$ along $S$, creating $R_{+}\left(\gamma^{\prime}\right)$ by adding $S_{+}^{\prime}$ to what is left of $R_{+}(\gamma)$ and creating $R_{-}\left(\gamma^{\prime}\right)$ by adding $S_{-}^{\prime}$ to what is left of $R_{-}(\gamma)$. Finally, one creates the annuli of $\gamma^{\prime}$ by "thickening" $R_{+}\left(\gamma^{\prime}\right) \cap R_{-}\left(\gamma^{\prime}\right)$.

Example 9.10 If $L \subset Y$ is a link and if $R$ is a Seifert surface of $L$ then there is a sutured manifold decomposition $Y(L) \rightsquigarrow{ }^{R} Y(R)$. Furthermore, if $L$ has $l$ components then there is a sutured manifold decomposition $Y(l) \rightsquigarrow{ }^{A} Y(L)$, where $A \subset Y(l)$ is a union of embedded annuli "around" the link $L$.

The following definition can be found in [2].

Definition 9.11 A sutured manifold decomposition $(M, \gamma) \rightsquigarrow D\left(M^{\prime}, \gamma^{\prime}\right)$ where $D$ is a disc properly embedded in $M$ and $|D \cap s(\gamma)|=2$ is called a product decomposition.

Remark 9.12 If $(M, \gamma)$ is balanced and if $(M, \gamma) \rightsquigarrow^{D}\left(M^{\prime}, \gamma^{\prime}\right)$ is a product decomposition then $\left(M^{\prime}, \gamma^{\prime}\right)$ is also balanced.

The following lemma will be very useful for computing sutured Floer homology since we can simplify the topology of our sutured manifold before computing the invariant.

Lemma 9.13 Let $(M, \gamma)$ be a balanced sutured manifold. If $(M, \gamma) \rightsquigarrow^{D}\left(M^{\prime}, \gamma^{\prime}\right)$ is a product decomposition then

$$
\operatorname{SFH}(M, \gamma)=\operatorname{SFH}\left(M^{\prime}, \gamma^{\prime}\right)
$$


Proof Let $N(D)$ be a regular neighborhood of $D$ and choose a diffeomorphism $t: N(D) \rightarrow[-1,4]^{3}$ mapping $D$ to $\{3 / 2\} \times[-1,4]^{2}$ and sending $s(\gamma) \cap N(D)$ to $[-1,4] \times \partial[-1,4] \times\{3 / 2\}$. Let $p_{3}:[-1,4]^{3} \rightarrow[-1,4]$ denote the projection onto the third factor. Then we can extend the function $p_{3} \circ t$ from $N(D)$ to a Morse function $f: M \rightarrow \mathbb{R}$ as described in the proof of Proposition 2.13. Note that $f$ has no critical points in $N(D)$ and that $D$ is a union of flowlines of $\operatorname{grad}(f)$ connecting $R_{-}(\gamma)$ with $R_{+}(\gamma)$. From $f$ we obtain a balanced diagram $(\Sigma, \boldsymbol{\alpha}, \boldsymbol{\beta})$ where $\Sigma=f^{-1}(3 / 2)$.

The $\operatorname{arc} \delta=D \cap \Sigma$ has boundary on $\partial \Sigma$ and is disjoint from $\boldsymbol{\alpha}$ and $\boldsymbol{\beta}$. Since $\partial \delta \subset \partial \Sigma$ every domain $\mathcal{D} \in D(\Sigma, \boldsymbol{\alpha}, \boldsymbol{\beta})$ has zero multiplicity in the domain containing $\delta$. Cutting $\Sigma$ open along $\delta$ we obtain a surface $\Sigma^{\prime}$. The balanced diagram $\left(\Sigma^{\prime}, \boldsymbol{\alpha}, \boldsymbol{\beta}\right)$ defines the sutured manifold $\left(M^{\prime}, \gamma^{\prime}\right)$. Using Proposition 3.15 isotope $\left(\Sigma^{\prime}, \boldsymbol{\alpha}, \boldsymbol{\beta}\right)$ to obtain an admissible diagram $\left(\Sigma^{\prime}, \boldsymbol{\alpha}, \boldsymbol{\beta}^{\prime}\right)$ of $\left(M^{\prime}, \gamma^{\prime}\right)$. Then $\left(\Sigma, \boldsymbol{\alpha}, \boldsymbol{\beta}^{\prime}\right)$ is an admissible diagram of $(M, \gamma)$ since every periodic domain $\mathcal{P} \neq 0$ has zero multiplicity in the domain containing $\delta$ and thus corresponds to a periodic domain in $\left(\Sigma^{\prime}, \boldsymbol{\alpha}, \boldsymbol{\beta}^{\prime}\right)$, so it has both positive and negative multiplicities. Thus we can suppose that both diagrams $(\Sigma, \boldsymbol{\alpha}, \boldsymbol{\beta})$ and $\left(\Sigma^{\prime}, \boldsymbol{\alpha}, \boldsymbol{\beta}\right)$ are admissible.

Since every domain $\mathcal{D} \in D(\Sigma, \boldsymbol{\alpha}, \boldsymbol{\beta})$ has zero multiplicity in the domain containing $\delta$, the chain complexes $C F(\Sigma, \boldsymbol{\alpha}, \boldsymbol{\beta})$ and $C F\left(\Sigma^{\prime}, \boldsymbol{\alpha}, \boldsymbol{\beta}\right)$ are isomorphic.

As an application we prove a generalization of Proposition 9.1.

Proposition 9.14 If $Y$ is a closed connected oriented 3-manifold then for all $n \geq 1$,

$$
\operatorname{SFH}(Y(n)) \approx \bigoplus_{2^{n-1}} \widehat{\mathrm{HF}}(Y) \approx \widehat{\mathrm{HF}}(Y) \otimes \bigotimes_{n-1} \mathbb{Z}^{2}
$$

Proof We prove the claim by induction on $n$. The case $n=1$ is true according to Proposition 9.1. Suppose that we know the proposition for some $n-1 \geq 1$. Then applying the induction hypotheses to $\left(Y \#\left(S^{1} \times S^{2}\right)\right)(n-1)$ we get that

$$
\operatorname{SFH}\left(\left(Y \#\left(S^{1} \times S^{2}\right)\right)(n-1)\right) \approx \widehat{\mathrm{HF}}\left(Y \#\left(S^{1} \times S^{2}\right)\right) \otimes \bigotimes_{n-2} \mathbb{Z}^{2} \approx \widehat{\mathrm{HF}}(Y) \otimes \bigotimes_{n-1} \mathbb{Z}^{2} .
$$

Here we used the connected sum formula $\widehat{\mathrm{HF}}\left(Y \#\left(S^{1} \times S^{2}\right)\right) \approx \widehat{\mathrm{HF}}(Y) \otimes \widehat{\mathrm{HF}}\left(S^{1} \times S^{2}\right)$ and the fact that $\widehat{\mathrm{HF}}\left(S^{1} \times S^{2}\right) \approx \mathbb{Z}^{2}$. On the other hand we will show that there is a product decomposition $Y(n-1) \#\left(S^{1} \times S^{2}\right) \rightsquigarrow^{D} Y(n)$, which shows together with Lemma 9.13 that the induction hypothesis is also true for $n$.

To find the product disc $D$ choose a ball $B_{1} \subset S^{1} \times S^{2}$ such that there is a point $p \in S^{1}$ for which $\{p\} \times S^{2}$ intersects $B_{1}$ in a disc. Then let $D$ be the closure 
of $\left(\{p\} \times S^{2}\right) \backslash B_{1}$. We can also choose a simple closed curve $s_{1} \subset \partial B_{1}$ so that $\left|s_{1} \cap D\right|=2$. Now construct $\left(Y \#\left(S^{1} \times S^{2}\right)\right)(n-1)$ as in Example 2.3 using $B_{1}$ and $s_{1}$ as above. Then $D$ is a product disc with the required properties.

Next we will generalize the above idea to obtain a connected sum formula.

Proposition 9.15 Let $(M, \gamma)$ and $(N, v)$ be balanced sutured manifolds and let $Y$ be a closed oriented 3-manifold. Then

$\begin{aligned} \operatorname{SFH}((M, \gamma) \#(N, \nu)) & =\operatorname{SFH}(M, \gamma) \otimes \operatorname{SFH}(M, v) \\ \text { Furthermore, } & \operatorname{SFH}(M \# Y, \gamma)=\operatorname{SFH}(M, \gamma) \otimes \widehat{\operatorname{HF}}(Y) .\end{aligned}$

Proof There are product decompositions

and

$$
(M, \gamma) \#(N, \nu) \rightsquigarrow^{D}(M, \gamma) \coprod N(1)
$$

$$
(M, \gamma) \# Y \rightsquigarrow^{D}(M, \gamma) \bigsqcup Y(1) \text {. }
$$

To see this push some part of the boundary of $M$ containing a segment of $\gamma$ into the connected sum tube using a finger move and repeat the idea described in the proof of Proposition 9.14 (also see Figure 2).

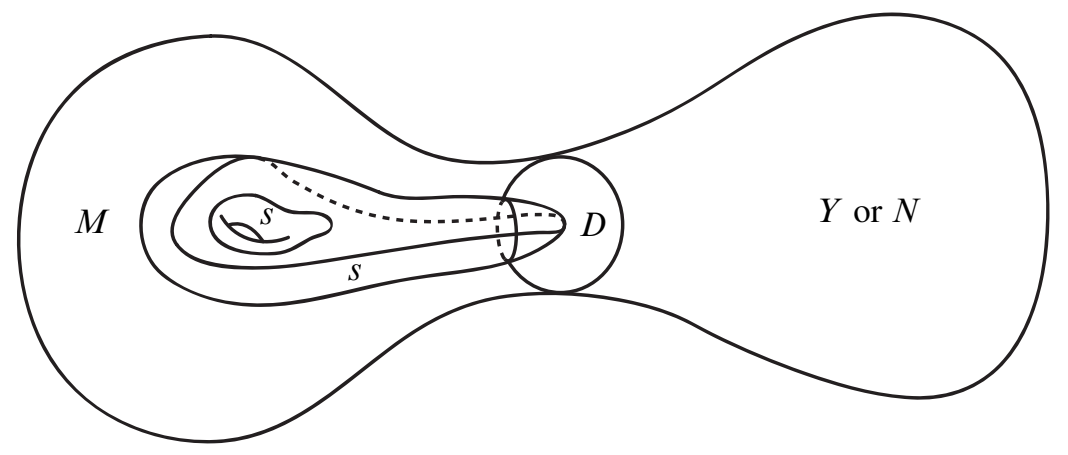

Figure 2: Product decomposition of a connected sum

This implies that

and

$$
\operatorname{SFH}((M, \gamma) \#(N, v))=\operatorname{SFH}(M, \gamma) \otimes \operatorname{SFH}(N(1))
$$

$$
\operatorname{SFH}(M \# Y, \gamma)=\operatorname{SFH}(M, \gamma) \otimes \operatorname{SFH}(Y(1)) \text {. }
$$

Proposition 9.1 says that $\operatorname{SFH}(Y(1))=\widehat{\mathrm{HF}}(Y)$. Since $N(1)=(N, v) \# S^{3}(1)$ we can apply (9-1) again and we get that

$$
\operatorname{SFH}(N(1))=\operatorname{SFH}(N, v) \otimes \operatorname{SFH}\left(S^{3}(2)\right) .
$$


From the existence of a product decomposition $\left(S^{1} \times S^{2}\right)(1) \rightsquigarrow S^{3}(2)$ (see Proposition 9.14) we obtain that

$$
\operatorname{SFH}\left(S^{3}(2)\right) \approx \widehat{\mathrm{HF}}\left(S^{1} \times S^{2}\right) \approx \mathbb{Z}^{2}
$$

Corollary 9.16 If $(M, \gamma)$ is a connected balanced sutured manifold and $n \geq 1$ then

$$
\operatorname{SFH}(M(n)) \approx \operatorname{SFH}(M, \gamma) \otimes \mathbb{Z}^{2^{n}} .
$$

Proof The claim follows by induction on $n$. The case $n=0$ is trivial. Now let us suppose that $n>0$. Since $M(n)=M(n-1) \# S^{3}(1)$ we get from Proposition 9.15 that $\operatorname{SFH}(M(n)) \approx \operatorname{SFH}(M(n-1)) \otimes \operatorname{SFH}\left(S^{3}(1)\right) \otimes \mathbb{Z}^{2}$. Here $\operatorname{SFH}\left(S^{3}(1)\right) \approx \widehat{\mathrm{HF}}\left(S^{3}\right) \approx \mathbb{Z}$. This concludes the proof.

Definition 9.17 A sutured manifold $(M, \gamma)$ is called taut if $M$ is irreducible and $R(\gamma)$ is incompressible and Thurston norm-minimizing in its homology class in $H_{2}(M, \gamma)$.

Proposition 9.18 Suppose that $(M, \gamma)$ is an irreducible balanced sutured manifold. If $(M, \gamma)$ is not taut then $\operatorname{SFH}(M, \gamma)=0$.

The following proof is due to $\mathrm{Yi} \mathrm{Ni.}$

Proof Since $(M, \gamma)$ is not taut and $M$ is irreducible either $R_{+}(\gamma)$ or $R_{-}(\gamma)$, say $R_{+}(\gamma)$, is either compressible or it is not Thurston norm minimizing in $H_{2}(M, \gamma)$. In both cases there exists a properly embedded surface $(S, \partial S) \subset(M, \gamma)$ such that $\chi(S)>\chi\left(R_{+}(\gamma)\right)$, no collection of components of $S$ is null-homologous and the class $[S, \partial S]=\left[R_{+}(\gamma), \partial R_{+}(\gamma)\right]$ in $H_{2}(M, \gamma)$. Then decomposing $(M, \gamma)$ along $S$ we get two connected sutured manifolds $\left(M_{+}, \gamma_{+}\right)$and $\left(M_{-}, \gamma_{-}\right)$. Here $R_{+}(\gamma)=R_{+}\left(\gamma_{+}\right)$ and $R_{-}(\gamma)=R_{-}\left(\gamma_{-}\right)$.

As in the proof of Proposition 2.13 construct Morse functions $f_{+}$and $f_{-}$on $M_{+}$and $M_{-}$, respectively, having no index zero and three critical points. Then $f=f_{+} \cup f_{-}$ is a Morse function on $M$ that has $S$ as a level surface. Denote by $C_{i}(h)$ the set and by $c_{i}(h)$ the number of index $i$ critical points of a Morse function $h$. Now rearrange $f$ by switching $C_{1}\left(f_{+}\right)$and $C_{2}\left(f_{-}\right)$to obtain a self-indexing Morse function $g$ (see Milnor [5]). Then $g$ induces a Heegaard diagram $\left(S^{\prime}, \boldsymbol{\alpha}_{+} \cup \boldsymbol{\alpha}_{-}, \boldsymbol{\beta}_{+} \cup \boldsymbol{\beta}_{-}\right)$, where $\boldsymbol{\alpha}_{ \pm}$ and $\boldsymbol{\beta}_{ \pm}$are the sets of attaching circles corresponding to the critical points in $C_{1}\left(f_{ \pm}\right)$ and $C_{2}\left(f_{ \pm}\right)$, respectively, and $S^{\prime}$ is obtained by performing $c_{1}\left(f_{+}\right)+c_{2}\left(f_{-}\right)$zero surgeries on $S$ whose belt circles are the elements of $\boldsymbol{\alpha}_{+} \cup \boldsymbol{\beta}_{-}$(see Figure 3). Our main observation is that $\alpha \cap \beta=\varnothing$ if $\alpha \in \boldsymbol{\alpha}_{+}$and $\beta \in \boldsymbol{\beta}_{-}$, because they are belt circles of two disjoint handles added to $S$. This property of the Heegaard diagram is 


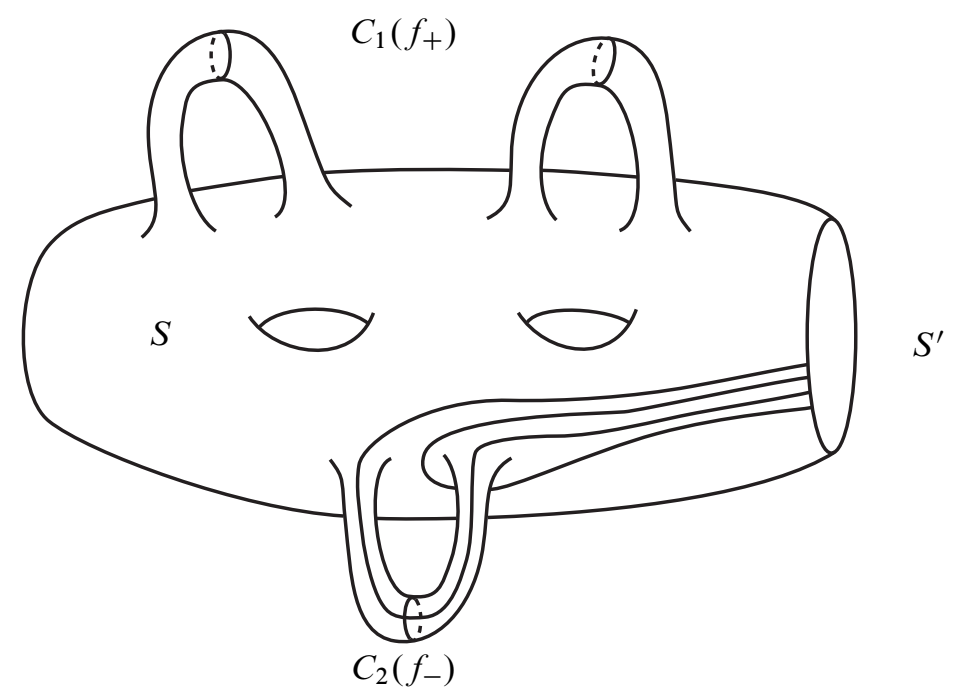

Figure 3: The surface $S^{\prime}$ with two $\boldsymbol{\alpha}_{+}$curves on the top, one $\boldsymbol{\beta}_{-}$curve on the bottom, and with two winding arcs, one of them intersecting $\boldsymbol{\beta}_{-}$and being disjoint from $\boldsymbol{\alpha}_{+}$

preserved if we apply the winding argument of Proposition 3.15 using winding arcs $\gamma_{1}, \ldots, \gamma_{l}$ that satisfy the following property: if $\gamma_{k} \cap \beta \neq \varnothing$ for $\beta \in \boldsymbol{\beta}_{-}$and $1 \leq k \leq l$ then $\gamma_{k} \cap \alpha=\varnothing$ for every $\alpha \in \alpha_{+}$. Such arcs $\gamma_{1}, \ldots, \gamma_{l}$ are easy to construct (see Figure 3 ). Thus we can assume our Heegaard diagram is admissible.

The only $\beta$ curves that can intersect $\alpha \in \boldsymbol{\alpha}_{+}$are the elements of $\boldsymbol{\beta}_{+}$. But

$$
0>\chi\left(R_{+}(\gamma)\right)-\chi(S)=2\left(c_{2}\left(f_{+}\right)-c_{1}\left(f_{+}\right)\right),
$$

thus $\left|\boldsymbol{\alpha}_{+}\right|=c_{1}\left(f_{+}\right)>c_{2}\left(f_{+}\right)=\left|\boldsymbol{\beta}_{+}\right|$. This shows that $\mathbb{T}_{\alpha} \cap \mathbb{\mathbb { T }}_{\beta}=\varnothing$ for this Heegaard diagram. Indeed, if there was a permutation $\pi \in S_{d}$ such that $\alpha_{i} \cap \beta_{\pi(i)} \neq \varnothing$ for every $1 \leq i \leq d$, then for $\alpha_{i} \in \boldsymbol{\alpha}_{+}$we would have $\beta_{\pi(i)} \in \boldsymbol{\beta}_{+}$, and the injectivity of $\pi$ would imply that $\left|\boldsymbol{\beta}_{+}\right| \geq\left|\boldsymbol{\alpha}_{+}\right|$.

Question 9.19 Is the converse of Proposition 9.18 true, ie, if $\operatorname{SFH}(M, \gamma)=0$ does it follow that $(M, \gamma)$ is not taut?

\section{Seifert surfaces}

Now we turn our attention to Example 2.6. These sutured manifolds are of particular interest to us due to the following theorem of Gabai [2, Theorem 1.9]. 
Theorem 10.1 Suppose that $R$ is an oriented surface in $S^{3}$ and let $L$ be the oriented link $\partial R$. Then $L$ is a fibred link with fibre $R$ if and only if $S^{3}(R)$ is a product sutured manifold.

This becomes interesting in light of the following conjecture.

Conjecture 10.2 Let $K$ be a knot in $S^{3}$ and let $R$ be a genus $g$ Seifert surface of $K$. Then $\widehat{\operatorname{HFK}}(K, g) \approx \operatorname{SFH}\left(S^{3}(R)\right)$.

Note that from Alexander duality we get that

$$
H_{2}\left(S^{3}(R) ; \mathbb{Z}\right) \approx \widetilde{H}^{0}(R \times I ; \mathbb{Z})=0
$$

Thus, together with a positive answer to Question 9.19, Conjecture 10.2 would give a new proof of the fact that $\widehat{\mathrm{HFK}}(K, g(K)) \neq 0$, where $g(K)$ denotes the threegenus of $K$. Combining Conjecture 10.2 with Proposition 9.18 we would get that $\widehat{\operatorname{HFK}}(K, g)=0$ for $g>g(K)$.

Finally, if we combine Theorem 10.1, Conjecture 10.2 and Question 9.7 we would obtain a proof of the following conjecture (see [10, Theorem 1.1] and [6]). Note that a fibred knot has a unique minimal genus Seifert surface up to isotopy.

Conjecture 10.3 Let $K$ be a knot in $S^{3}$. Then $K$ is fibred if and only if

$$
\widehat{\operatorname{HFK}}(K, g(K)) \approx \mathbb{Z} \text {. }
$$

In what follows we collect some evidence supporting Conjecture 10.2. First we recall a result of Hedden [3].

Proposition 10.4 Let $K$ be knot in $S^{3}$ and let $D_{+}(K, t)$ denote the positive $t$-twisted Whitehead double of $K$. The meridian $\mu \subset S^{3}$ of $K$ can be viewed as a knot in $S_{t}^{3}(K)$ (the parameter $t$ Dehn surgery on $K$ ). Then we have

$$
\widehat{\operatorname{HFK}}\left(D_{+}(K, t), 1\right) \approx \widehat{\operatorname{HFK}}\left(S_{t}^{3}(K), \mu\right)
$$

Using this result we can prove the following.

Theorem 10.5 Let $R$ be the Seifert surface of $D_{+}(K, t)$ obtained by taking the satellite of the surface $R_{2 t}$ defined in Proposition 10.6 (see below). Then

$$
\widehat{\operatorname{HFK}}\left(D_{+}(K, t), 1\right) \approx \operatorname{SFH}\left(S^{3}(R)\right) .
$$


Note that the Seifert genus of $D_{+}(K, t)$ is 1 . The left hand side of Figure 2 shows $D_{+}(U, 1)$ together with its natural Seifert surface. The surface $R$ is obtained by taking a solid torus neighborhood of $U$ containing $R_{2 t}$ and wrapping it around $K$ using the Seifert framing of $K$.

Proof In light of Proposition 10.4 we only have to show that

$$
\operatorname{SFH}\left(S^{3}(R)\right) \approx \widehat{\operatorname{HFK}}\left(S_{t}^{3}(K), \mu\right) .
$$

Let $K_{2,2 t}$ denote the $(2,2 t)$-cable of $K$ (which is a two component link) and let $R^{\prime}$ be the natural Seifert surface of $K_{2,2 t}$. Then there is a product decomposition $S^{3}(R) \rightsquigarrow^{D} S^{3}\left(R^{\prime}\right)$ (see Figure 4). This does not change the sutured Floer homology according to Lemma 9.13. Now we can apply Remark 9.3 to compute $\operatorname{SFH}\left(S^{3}\left(R^{\prime}\right)\right)$. If we glue $S^{1} \times D^{2}$ to $S^{3}\left(R^{\prime}\right)$ the meridian $\{1\} \times \partial D^{2}$ maps to one component of $K_{2,2 t}$ and we can suppose that the longitude $S^{1} \times\{1\}$ maps to the meridian $\mu$ of the original knot $K$. After gluing in $S^{1} \times D^{2}$ we obtain $S_{t}^{3}(K)$. Note that in $S_{t}^{3}(K)$ the knot $S^{1} \times\{0\}$ is isotopic to $\mu$ since the longitude of $S^{1} \times D^{2}$ was identified with $\mu$. Thus

$$
\operatorname{SFH}\left(S^{3}\left(R^{\prime}\right)\right) \approx \widehat{\operatorname{HFK}}\left(S_{t}^{3}(K), \mu\right)
$$

which concludes the proof.

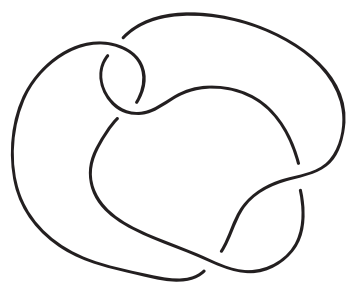

$\mathrm{Tw}_{2}$

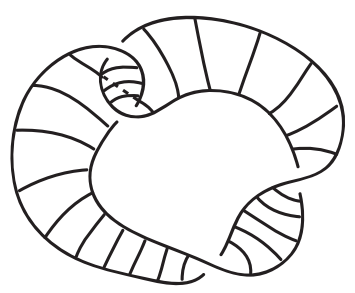

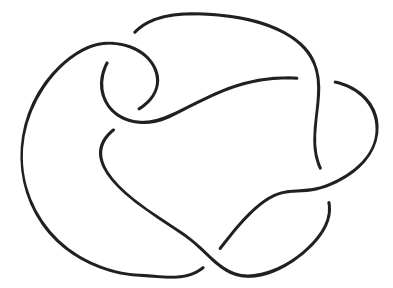

$\mathrm{Tw}_{3}$

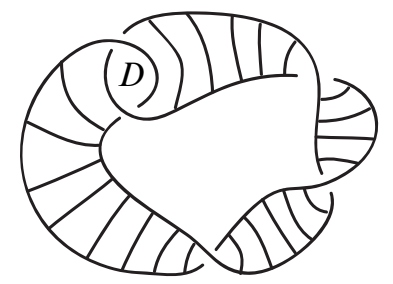

Figure 4: Standard diagrams of the knots $\mathrm{Tw}_{2}=D_{+}(U, 1)$ and $\mathrm{Tw}_{3}$ together with the Seifert surfaces $R_{2}$ and $R_{3}$ obtained using the Seifert algorithm

The following similar statement can be proved without making use of Proposition 10.4. 
Proposition 10.6 Let $\mathrm{Tw}_{n}$ denote the standard diagram of the twist knot with $n$ half right-handed twists and let $R_{n}$ be the genus one Seifert surface of $\mathrm{Tw}_{n}$ obtained from the Seifert algorithm (see Figure 4). Then

$$
\operatorname{SFH}\left(S^{3}\left(R_{n}\right)\right) \approx \widehat{\operatorname{HFK}}\left(\operatorname{Tw}_{n}, 1\right) \approx \mathbb{Z}^{[(n+1) / 2]} .
$$

Proof Let $R_{n}^{\prime}$ be the unique Seifert surface of the torus link $T_{2,2[(n+1) / 2]}$. Then there is a product decomposition $S^{3}\left(R_{n}\right) \rightsquigarrow^{D} S^{3}\left(R_{n}^{\prime}\right)$. As in the proof of Theorem 10.5 we have an isomorphism

$$
\operatorname{SFH}\left(S^{3}\left(R_{n}^{\prime}\right)\right) \approx \widehat{\mathrm{HFK}}\left(S_{[(n+1) / 2]}^{3}(U), \mu\right) .
$$

But $S_{[(n+1) / 2]}^{3}(U)$ is homeomorphic to the lens space $L([(n+1) / 2], 1)$. Thus, according to [11],

$$
\operatorname{SFH}\left(S^{3}\left(R_{n}\right)\right) \approx \widehat{\operatorname{HFK}}(L([(n+1) / 2]), \mu) \approx \mathbb{Z}^{[(n+1) / 2]} .
$$

Since $\mathrm{Tw}_{n}$ is alternating, $\operatorname{rk}\left(\widehat{\mathrm{HFK}}\left(\mathrm{Tw}_{n}, 1\right)\right)$ agrees with the absolute value of the leading coefficient of the Alexander polynomial of $K$, which is $[(n+1) / 2]$.

Finally one more evidence supporting Conjecture 10.2.

Proposition 10.7 Suppose that the knot $K$ has at most 7 crossings and that $K \neq 7_{4}$. Then $K$ has a unique minimal genus Seifert surface $R$ and

$$
\widehat{\operatorname{HFK}}(K, g(K)) \approx \operatorname{SFH}\left(S^{3}(R)\right) .
$$

Proof The fact that $K$ has a unique minimal genus Seifert surface was proved by Kobayashi [4]. We already know the statement for fibred knots. The only nonfibred at most 7 crossing knots are $5_{2}, 6_{1}, 7_{2}, 7_{3}, 7_{4}$ and $7_{5}$. The knots $5_{2}, 6_{1}$ and $7_{2}$ are twist knots and hence the result follows from Proposition 10.6. The case of $7_{3}$ and $7_{5}$ is analogous, we can reduce the computation of $\operatorname{SFH}\left(S^{3}(R)\right)$ using product decompositions to computing knot Floer homology of knots in lens spaces. Both knots are alternating, so their knot Floer homology can be computed from the Alexander polynomial.

Remark 10.8 By understanding how a balanced diagram changes under a disc decomposition of the underlying sutured manifold we could prove the following formula. If the oriented surface $R \subset S^{3}$ is the Murasugi sum of the surfaces $R_{1}$ and $R_{2}$ then over any field $\mathbb{F}$

$$
\operatorname{SFH}\left(S^{3}(R) ; \mathbb{F}\right) \approx \operatorname{SFH}\left(S^{3}\left(R_{1}\right) ; \mathbb{F}\right) \otimes \operatorname{SFH}\left(S^{3}\left(R_{2}\right) ; \mathbb{F}\right) .
$$


This formula is analogous to the Murasugi sum formula of [6].

The knot 74 has two distinct minimal genus Seifert surfaces, both of them Murasugi sums of two embedded annuli. In both cases we get that the sutured Floer homology associated to the Seifert surface is isomorphic to $\mathbb{Z}^{4}$, supporting Conjecture 10.2. I will deal with these results in a separate paper.

\section{References}

[1] D Gabai, Foliations and the topology of 3-manifolds, J. Differential Geom. 18 (1983) 445-503 MR723813

[2] D Gabai, Detecting fibred links in $S^{3}$, Comment. Math. Helv. 61 (1986) 519-555 MR870705

[3] M Hedden, Knot Floer homology of Whitehead doubles arXiv:math.GT/0606094

[4] T Kobayashi, Uniqueness of minimal genus Seifert surfaces for links, Topology Appl. 33 (1989) 265-279 MR1026928

[5] J Milnor, Lectures on the $h$-cobordism theorem, Notes by L. Siebenmann and J. Sondow, Princeton University Press, Princeton, N.J. (1965) MR0190942

[6] Y Ni, Sutured Heegaard diagrams for knots, Algebr. Geom. Topol. 6 (2006) 513-537 MR2220687

[7] P Ozsváth, Z Szabó, Holomorphic disks, link invariants, and the multi-variable Alexander polynomial arXiv:math.GT/0512286

[8] P Ozsváth, Z Szabó, Holomorphic disks and knot invariants, Adv. Math. 186 (2004) 58-116 MR2065507

[9] P Ozsváth, Z Szabó, Holomorphic disks and topological invariants for closed threemanifolds, Ann. of Math. (2) 159 (2004) 1027-1158 MR2113019

[10] P Ozsváth, Z Szabó, Heegaard Floer homology and contact structures, Duke Math. J. 129 (2005) 39-61 MR2153455

[11] P Ozsváth, Z Szabó, On knot Floer homology and lens space surgeries, Topology 44 (2005) 1281-1300 MR2168576

[12] J A Rasmussen, Floer homology and knot complements, PhD thesis, Harvard University (2003)

Department of Mathematics, Princeton University

Princeton, NJ 08544, USA

ajuhasz@math.princeton.edu

Received: 18 March 06

Algebraic 83 Geometric Topology, Volume 6 (2006) 